\title{
Systematic characterization of low-pressure capacitively coupled
} hydrogen discharges

\author{
A. Salabas, ${ }^{\text {a) }}$ L. Marques, ${ }^{\text {a) }}$ J. Jolly, ${ }^{\text {b) }}$ G. Gousset, ${ }^{\text {c) }}$ and L.L. Alves ${ }^{\text {a),d) }}$ \\ a) Centro de Física dos Plasmas, Instituto Superior Técnico, 1049-001 Lisboa, Portugal \\ b) Laboratoire de Physique et Technologie des Plasmas, École Polytechnique, \\ 91128 Palaiseau Cedex, France \\ c) Laboratoire de Physique des Gaz et des Plasmas, Université de Paris-Sud, \\ 91405 Orsay Cedex, France \\ (Received
}

d)Electronic mail: llalves@alfa.ist.utl.pt 


\begin{abstract}
The paper presents a systematic characterization of pure hydrogen capacitively coupled discharges, produced in a parallel plate cylindrical setup. A two-dimensional, time-dependent fluid model is used to describe the production, transport and destruction of electrons and positive ions $\mathrm{H}^{+}, \mathrm{H}_{2}^{+}$, and $\mathrm{H}_{3}^{+}$, at different frequencies $(13.56-60 \mathrm{MHz})$, pressures $(0.2-8$ Torr $)$, rf applied voltages $(50-450 \mathrm{~V})$ and geometric dimensions $(1.6-12.8 \mathrm{~cm}$ radii and $1.6-6.4 \mathrm{~cm}$ interelectrode distances). A good agreement is found between calculation results and experimental measurements for the coupled electrical power, the plasma potential and the self-bias potential, at various frequencies and rf applied voltages. However, the model generally underestimates the electron density with respect to its measured values. The paper discusses different space-time events, as the development of double ionization structures or the occurrence of field inversion and field reversal phenomena. The dependencies on pressure and frequency of the time-average electric field distribution are analysed and related to the electron displacement within space-charge sheaths. This study is later used to understand the variations of the hydrogen dissociation rate, with changes in discharge operating conditions. The influence of reactor dimensions in the spatial profiles of the plasma potential, the rf electric field, the electron density and the electron mean energy are analysed in terms of discharge symmetry. An investigation of the space-time averaged rf electric field variations, with changes in the applied voltage, pressure, and geometric dimensions is carried out. These variations are shown to follow an universal similarity curve, if an adequate normalization is used when plotting the rf electric field as a function of pressure. This innovative representation of rf discharges allows a univocal definition of a reactor working point, for given operating conditions.
\end{abstract}

PACS numbers: 52.50.Dg, 52.80.Pi, 52.50.Qt, 52.40.Kh, 52.65.Kj 


\section{INTRODUCTION}

Parallel plate capacitively coupled radio frequency (ccrf) discharges are of great interest in plasma assisted material processing applications, for example in the production of silicon and diamond using hydrogen based mixtures. This work is part of an effort to model and optimize an existing plasma enhanced chemical vapor deposition (pecvd) reactor, used for the production of hydrogenated microcristallin silicon ( $\mu$-Si:H) from $\mathrm{SiH}_{4}-\mathrm{H}_{2}$ gas mixtures, under high dilution conditions for silane. Our goal is to improve the characterization of this type of discharges, by analysing the variation of their main features with changes in operation conditions: frequency, pressure, applied voltage and reactor dimensions.

The pecvd reactor under study has a configuration similar to the GEC reference Cell ${ }^{1}$ (i.e. a parallel plate cylindrical setup with standard $6.4 \mathrm{~cm}$ radius and $\simeq 3.2 \mathrm{~cm}$ interelectrode distance), where a lateral grid has been used in order to confine the plasma between the electrodes. The paper presents a systematic characterization of pure hydrogen discharges produced with this device, at different frequencies $(13.56-60 \mathrm{MHz})$, pressures $(0.2-8$ Torr), rf applied voltages $(50-450 \mathrm{~V})$ and geometric dimensions $(1.6-12.8 \mathrm{~cm}$ radii and $1.6-6.4 \mathrm{~cm}$ interelectrode distances). The study is carried out by using a two-dimensional (2D) timedependent fluid model that describes the production, transport and destruction of electrons and positive ions $\mathrm{H}^{+}, \mathrm{H}_{2}^{+}$, and $\mathrm{H}_{3}^{+}$.

Model results are compared with experimental measurements for the electron density, the coupled electrical power, the plasma potential and the self-bias potential, for various frequencies and rf applied voltages. The evolution in time of the electric field distribution, and its time-average dependence on pressure and frequency are analysed. The influence of reactor dimensions in the spatial profiles of the plasma potential, the rf electric field, the electron density and the electron mean energy are also analysed. An investigation of the space-time averaged rf electric field variations with changes in the applied voltage, pressure, and geometric dimensions is carried out. These variations are shown to follow an universal similarity curve, if an adequate normalization is used when plotting the rf electric field as a function of pressure. Finally, the paper also discusses the influence of pressure and frequency on the hydrogen dissociation rate. 


\section{FORMULATION AND SOLUTION}

The transport of charged particles in ccrf discharges is described here using a fluid approach, by solving the continuity and momentum transfer equations for electrons, and $\mathrm{H}^{+}$, $\mathrm{H}_{2}^{+}$and $\mathrm{H}_{3}^{+}$positive ions, coupled with Poisson's equation and the electron mean energy transport equations. Boundary conditions involve symmetry considerations at reactor axis, and the imposition of the different particle and energy fluxes, together with the applied rf potential, at each physical boundary (electrode, wall). Details on the model and its numerical solution can be found in references. ${ }^{2,3}$

Model equations are solved given the applied voltage $V_{\mathrm{rf}}$ and frequency $f=1 / T_{\mathrm{rf}}\left(T_{\mathrm{rf}}\right.$ is the rf time period), the gas density $N\left(N=p / k_{B} T_{g}\right.$, where $p$ and $T_{g}$ are the gas pressure and temperature, respectively, and $k_{B}$ is the Boltzmann constant), and the geometric dimensions of the cylindrical reactor (the radius $R$ and the interelectrode distance $d$ ), yielding the spacetime profiles $(r, z, t)$ of charged particle densities and fluxes $\left[n_{e}, n_{i}, \vec{\Gamma}_{e}\right.$ and $\vec{\Gamma}_{i}$, where the subscripts $e$ and $i$ refer to electrons and ions, respectively], of the electron mean energy $\varepsilon$ and

its flux $\vec{\Gamma}_{\varepsilon}$, and the rf electric field $\vec{E}$. Model calculations are restricted to the volume between electrodes, corresponding to a 2D work-space delimited by the discharge axis $(r=0)$, the lateral grid $(r=R)$, the driven electrode $(z=0)$ and the grounded electrode $(z=d)$.

In the case of an asymmetric configuration reactor, with more surfaces grounded than driven, a pure capacitive mode operation requires the development of an offset potential, termed self-bias voltage ${ }^{2} V_{\mathrm{dc}}$, which is self-consistently calculated by the model. From simulation results it is also possible to evaluate the effective electrical power coupled to the plasma $^{2}$

$$
W_{\text {eff }} \equiv \frac{1}{T_{\text {rf }}} \int_{0}^{T_{\mathrm{rf}}}\left[V_{\mathrm{dc}}+V_{\mathrm{rf}} \cos (2 \pi f t)\right] I_{t}(t) d t,
$$

where $I_{t}$ represents the total current flowing to the driven electrode, obtained by integrating the axial component of total current density over the surface of the electrode

$$
\begin{aligned}
I_{t}(t) & =\int_{0}^{R}\left[J_{C_{z}}(r, 0, t)+J_{D_{z}}(r, 0, t)\right] 2 \pi r d r \\
J_{C_{z}} & =e\left(\sum_{i} \Gamma_{i_{z}}-\Gamma_{e_{z}}\right) \\
J_{D_{z}} & =\varepsilon_{0} \frac{\partial E_{z}}{\partial t} .
\end{aligned}
$$

In these equations, $J_{C_{z}}$ and $J_{D_{z}}$ represent, respectively, the axial conduction and displace- 
ment components of the total current density, $e$ is the electron charge and $\varepsilon_{0}$ is the vacuum permittivity.

The model is applied to ccrf discharges in pure hydrogen, assuming a constant pressure and temperature $\left(T_{g}=300 \mathrm{~K}\right)$. The paper uses the simplified kinetic scheme presented in Table I, where the different charged particle production and destruction mechanisms are summarized. We have used here constant (i.e. energy independent) branching ratios to discriminate between the ionization channels producing $\mathrm{H}_{2}^{+}$and $\mathrm{H}^{+}$, instead of using different ionization cross-sections for these mechanisms. Simulation tests show ${ }^{12}$ that the main consequence in using the latter alternative is a strong reduction in the population of $\mathrm{H}^{+}$. The work adopts the electron cross-sections compiled in Refs. 5-7, considering collisions only with ground state neutrals to yield electronic (including ionization and dissociation), vibrational and rotational excitations.

Electron transport parameters are calculated adopting the local mean energy approximation, ${ }^{2}$ which assumes that the space-time dependence of the electron energy distribution function $(\mathrm{EEDF})$ and its related transport parameters proceeds via the electron mean energy. The latter has a space-time profile given from the solution to the fluid code, being related to the EEDF by solving the electron Boltzmann equation (in a homogeneous and stationary, two-term approximation). The work adopts the low-field ion mobilities proposed in Refs. $4,13,14$, with a high-field dependence which follows either a $-1 / 2$ power law $^{9,15}$ for $\mathrm{H}^{+}$and $\mathrm{H}_{3}^{+}$ions, or the expression proposed in Ref. 16 for $\mathrm{H}_{2}^{+}$ions.

Model equations are discretized using a second order finite difference representation that includes boundary conditions. The model is solved in either a $32 \times 32$ or a $65 \times 65$ points grid, for typical $10^{3}$ time steps within each rf period. It is assumed that the steady-state solution is reached if the relative changes of various discharge parameters, between two consecutive periods, is less than $10^{-3}$. Typically, it takes less that $100 \mathrm{rf}$ cycles to meet this convergence criterion, which is applied to the particle densities, the electron mean energy, the plasma potential and the dc self-bias voltage. 


\section{RESULTS AND DISCUSSION}

\section{A. General characterization}

Simulations yield typical time-average 2D profiles for the electron density $\left(\bar{n}_{e}\right)$ and mean energy $(\bar{\varepsilon})$, as shown in Figs. 1(a)-(b) obtained at $f=13.56 \mathrm{MHz}, V_{\mathrm{rf}}=200 \mathrm{~V}, p=0.5$ Torr, and reactor dimensions $R=6.4 \mathrm{~cm}$ and $d=3.2 \mathrm{~cm}$. The profile of $\bar{n}_{e}$ exhibits an out-of-axis peak, located at half distance between electrodes, and very steep gradients in the sheath regions due to the electric confinement produced by the rf field. The profile of $\bar{\varepsilon}$ presents a maximum near the corner where the rf electrode meets the grounded grid, due to the strong intensity of the $\mathrm{rf}$ field in this region. The maxima of $\bar{n}_{e}$ and $\bar{\varepsilon}$ are located at different positions in the reactor, which illustrates the very high non-local effects associated with energy transport at this pressure.

For the same work conditions as before, Figs. 2(a)-(c) plot the time-average 2D density profiles of $\mathrm{H}^{+}, \mathrm{H}_{2}^{+}$and $\mathrm{H}_{3}^{+}$ions, respectively. From this figure one concludes that $\mathrm{H}_{3}^{+}$is the dominant ion, its maximum density being more than one order of magnitude higher than the mean densities of either $\mathrm{H}_{2}^{+}$or $\mathrm{H}^{+}$. This result is a direct consequence of the very efficient conversion of $\mathrm{H}_{2}^{+}$ions (produced from the ionization of hydrogen molecules by electron impact) into $\mathrm{H}_{3}^{+}$ions, by non-resonant charge exchange collisions $\mathrm{H}_{2}^{+}+\mathrm{H}_{2} \rightarrow \mathrm{H}_{3}^{+}+$ $\mathrm{H}+1.71 \mathrm{eV}$. Note that although this process becomes less efficient for $\mathrm{H}_{2}^{+}$energies above $10 \mathrm{eV}$ (as collisions with hydrogen molecules are then symmetric charge transfer processes), the model assumes the same efficiency for this reaction across the whole reactor. Hence, one can anticipate an overestimation of the $\mathrm{H}_{3}^{+}$density, and a subsequent underestimation of the $\mathrm{H}_{2}^{+}$density, within sheath regions.

An observation of Figs. 2(a)-(c) shows that the time-average spatial profiles of $\mathrm{H}_{3}^{+}$and $\mathrm{H}^{+}$densities present the same kind of features already observed in Fig. 1(a). However, $\mathrm{H}_{2}^{+}$ density does not follow this typical profile structure, with a single peak located at halfdistance between electrodes, presenting instead two maxima located at the vicinity of the reactor corners (where the grid meets either the upper or the lower electrodes). The reason for this alternative profile is related to the rapid conversion of $\mathrm{H}_{2}^{+}$into $\mathrm{H}_{3}^{+}$, which strongly limits the transport of the former across the discharge volume. Therefore, one can expect the spatial profile of $\mathrm{H}_{2}^{+}$density to give an accurate indication of the regions where this 
ion is actually produced, which also means that its profile should closely follow that of the time-average ionization rate of hydrogen.

The latter prediction can be confirmed from Fig. 3(a) which represents the time-averaged spatial contour plot of the hydrogen ionization rate. As expected, this rate presents two peaks located at positions similar to those of the $\mathrm{H}_{2}^{+}$density. Note that these maxima do not coincide with neither the maximum of the time-average electron density nor that of the electron mean energy, being located instead near positions where the time-average electron energy density $\overline{n_{e} \varepsilon}$ is higher, for which it is still possible to find enough electrons with relatively high energies. Notice that most of the ionization processes occur at the bulk-sheath interface, where the ionization rate is at least one order of magnitude above its bulk value. Notice further that the highest ionization peak can be found in front of the rf electrode, where the sheath potential drop is also higher.

We now discuss the space-time variation, at $r=0$ and during one $\mathrm{rf}$ period, of the hydrogen ionization rate, by analysing the development of each of the four peaks shown in Fig. 3(b). At both the beginning and the end of the rf period, the field barrier in front of the rf electrode becomes weaker, leading to an increase of the electron flux towards the electrode. Consequently, those electrons entering the rf sheath have enough energy to ionize the background gas, thus producing the first peaks located at $z \simeq 0.4 \mathrm{~cm}$ and $t \simeq 0$ or $t \simeq 70-74 \mathrm{~ns}$. Later, electrons located at the $\mathrm{rf}$ sheath entrance are heated by the increasing electric field in this region and, while they are pushed back into the bulk, they are able to produce a second ionization peak (more intense than the first one), located at $z \simeq 0.8 \mathrm{~cm}$ and $t \simeq 25 \mathrm{~ns}$. Since we are not considering here the production of secondary electrons following the ion bombardment of electrodes, the ionization collisions involving primary electrons reflected by the sheath are crucial for discharge maintenance. The predicted value for the maximum ionization rate is about $3 \times 10^{15} \mathrm{~cm}^{-3} \mathrm{~s}^{-1}$. Simultaneously with the increase of the rf electric field, it occurs a decrease of the electric field in the opposite sheath. Thus, as we approach half period, the outward flux of electrons located near the grounded electrode is intensified and a third ionization peak is observed at $z \simeq 2.8 \mathrm{~cm}$ and $t \simeq 28 \mathrm{~ns}$. Later on, and similarly to what happens within the rf sheath, the electron population very close to the grounded electrode is subject to Joule heating, due to an increase of the electric field in this region (after $t=37 \mathrm{~ns}$ ). Thus, at $z \simeq 2.4 \mathrm{~cm}$ and $t \simeq 65 \mathrm{~ns}$, a fourth ionization peak is observed, produced by the electron acceleration as the grounded sheath expands. As 
final observations one should note that: (i) the ionization processes produced in opposite sheaths, by the outward (towards the electrode) and inward (towards the discharge bulk) electron flows, occur almost simultaneously; (ii) for the same sheath, a time interval of at least $35 \mathrm{~ns}$ separates the two ionization peaks, which are located at different distances from the electrode. As an example for the rf sheath, the ionization peak due to electron heating occurs close to the bulk-sheath interface at $z \simeq 0.8 \mathrm{~cm}$, whereas the ionization peak due to electron diffusion is located at $z \simeq 0.4 \mathrm{~cm}$. The development of double ionization structures in front of the electrodes has been predicted by other fluid and Particle-in-Cell/Monte-Carlo models for hydrogen discharges, ${ }^{17}$ and it appears to be strongly dependent on the mobility of charged particles. For example, the space-time representation of the ionization rate for helium (having a higher electron-neutral elastic collision cross-section, hence lower electron mobility values when compared to hydrogen) shows no such double structure. ${ }^{3,18}$ Space-time resolved excitation measurements in hydrogen rf discharges monitor this kind of double structures using the Balmer-alpha excitation ${ }^{18}$ (transition $3 \mathrm{p}-2 \mathrm{~s}$, at wavelength $653.3 \mathrm{~nm}$ ).

\section{B. Electric field distribution. Field inversion and field reversal}

Figures 4(a)-(b) present a typical distribution of the axial electric field component $E_{z}$, at different times, for a hydrogen ccrf discharge operating at $f=13.56 \mathrm{MHz}, V_{\mathrm{rf}}=200 \mathrm{~V}$, $p=0.5$ Torr and the same reactor dimensions of Sec. III A. As expected, this distribution features a zero electric field region at discharge center and two high intensity electric field regions at discharge sheaths.

Focusing on the rf electrode sheath, one observes that in general $E_{z}$ is always negative near $z=0$ (thus oriented towards the electrode) due to the development of a negative self-bias voltage. However, during the time period when the magnitude of the electric field decreases [between $t=5 T_{\mathrm{rf}} / 10$ and $t=9 T_{\mathrm{rf}} / 10$, see Fig. $4(\mathrm{~b})$ ], we can observe the formation of a small positive value region for $E_{z}$, near the rf electrode. The massive presence of positive charges in the sheath and the electron accumulation at sheath border are responsible for the positive values of $E_{z}$ (at $t=8 T_{\mathrm{rf}} / 10$ and $t=9 T_{\mathrm{rf}} / 10$, for example). As the flow of electrons towards the rf electrode increases, and more electrons are allowed to enter the sheath, the local charge separation vanishes and the intensity of this $E_{z}$ positive value region decreases.

Later, at the early beginning of next period and before the rf sheath starts its expansion 
[see Fig. 4(a)], when the value of the (still positive) rf applied potential decreases, a second (very weak) space-charge barrier can develop to further limit the entrance of electrons in sheath region. Consequently, the field near the rf electrode shifts negative (the so-called field inversion) for axial positions just after its positive value region. The formation of this double sheath is depicted in Fig. 5, obtained at time $t=T_{\mathrm{rf}} / 20$ for hydrogen ccrf discharges operating in the same frequency and pressure conditions as before, at applied voltages of $200 \mathrm{~V}$ and $400 \mathrm{~V}$ (the use of a higher $V_{\mathrm{rf}}$ allows an amplification of this phenomenon). From this figure one observes that the values of $E_{z}$ alternate twice between negative and positive from $z=0$ to $z \simeq 2.4 \mathrm{~cm}$ (the latter position corresponding to the grounded sheath threshold).

Simulations performed in hydrogen under conditions favoring the development of strong electric fields in sheath regions (e.g., adopting constant ion mobilities at $f=13.56 \mathrm{MHz}$, $p=0.35$ Torr and $V_{\mathrm{rf}}=350 \mathrm{~V}$, thus enhancing the flow of ions towards the sheaths) lead to a so-called field reversal situation near the rf electrode. This other phenomenon is associated to the enlargement of the formerly observed $E_{z}$ positive value region, for a very short time interval between $t \simeq 9 T_{\mathrm{rf}} / 10$ and $t=T_{\mathrm{rf}}$, during which the instantaneous orientation of the $E_{z}$ field becomes directed towards the discharge bulk (even at the rf electrode!). This effect comes as a response to the enhanced ion flow towards the rf electrode, and is rapidly canceled by the decrease of the rf potential which finally reverses the field direction towards the rf electrode, once again. The field reversal phenomenon has been experimentally observed in hydrogen discharges, ${ }^{18}$ and is often used to account for the intense $\mathrm{H}_{2}$ excitation near the rf electrode. The fact that current fluid simulations can only predict a field reversal under very particular (and extreme) operating conditions probably indicates that the transport description of hydrogen ions within rf sheaths is to be improved still.

\section{Influence of frequency and applied voltage. Comparison with experiment}

The production of $\mu$-Si:H thin films using typical pecvd operation conditions (a precursor mixture of $\mathrm{SiH}_{4}-\mathrm{H}_{2}$ under high dilution conditions for silane, at $f=13.56 \mathrm{MHz}$, $V_{\mathrm{rf}} \simeq 100-200 \mathrm{~V}$ and $p \simeq 300-500$ mTorr), leads to deposition rates $3-5$ times lower than the ones characterizing the formation of hydrogenated amorphous silicon films, using the same technique. In order to circumvent this important limitation in the production 
of microcristallin structures, several experimental ${ }^{19,20}$ and computational ${ }^{21-23}$ studies have proposed to increase the reactor operation frequencies, has a mean to enhance the density of electrons without affecting the energy of the ion population, which plays a major role in controlling film properties. In this way, it would be possible not only to increase the dissociation rates of both silane and hydrogen (and consequently to increase the thin film deposition rates), but also to ensure a higher flow of non-energetic hydrogen ions towards the substrate (thus favoring the formation of a microcristallin structure).

Figure 6 represents the variation of the time-average electron density with the rf applied voltage, at discharge axis and half-way between electrodes $\left[\bar{n}_{e}(0, d / 2)\right]$, for pressure $p=0.3$ Torr, reactor dimensions $R=6.4 \mathrm{~cm}$ and $d=3.0 \mathrm{~cm}$, and multiple operation frequencies of $13.56 \mathrm{MHz}, 27.12 \mathrm{MHz}$ and $40.68 \mathrm{MHz}$. As observed, the electron density increases with both $V_{\text {rf }}$ and $f$ as a consequence of the enhanced energy transfer between the rf electric field and the electron plasma population. Figure 6 also compares simulation results with experimental measurements obtained using a planar Langmuir probe. ${ }^{12}$ Although a reasonable qualitative agreement is found between simulations and experiment, the values of the calculated electron densities are a factor $3-7$ bellow the measured ones, and this difference is aggravated at both low applied voltages and frequencies (note that the experimental points in Fig. 6 were divided by a factor 3). The observed disagreement is most probably associated to a combination of factors: the incomplete description of the discharge space-charge sheaths (for example, due to the absence of the non-linear inertia term in the electron flux equation ${ }^{2}$ ), and the simplified hydrogen kinetics considered here.

At low frequencies the influence of discharge sheaths spreads across the entire discharge volume, as can be confirmed by analysing Fig. 7 representing the time-average distributions of the axial rf electric field at discharge axis, for the different frequency values considered here. From this figure one observes an enlargement of the zero-field region at discharge center as $f$ increases, simultaneously with a compression of sheaths where the rf electric field is enhanced. In fact, as frequency increases, the ability of electrons to follow the field oscillations is strongly limited, which leads to higher electric fields near discharge boundaries in order to ensure an effective control of space-charge separation over smaller time periods, and simultaneously to prevent major electron wall losses. The increase of the electric field within discharge sheaths has the following major consequences: (i) a reduction of sheath thickness and a discharge asymmetrization; (ii) an enhancement of the ionization rate leading 
to higher electron densities (see Fig. 6); (iii) an increase of the effective electrical power coupled to the plasma [see Fig. 8(a)], due to an enhancement of the total current flowing to the driven electrode.

Figure $8(\mathrm{a})$ plots $W_{\text {eff }}$ as a function of $V_{\text {rf }}$ for the different frequency values considered here. As expected, the coupled electrical power increases with both rf applied voltage and frequency, as a direct result of more intense total voltage and currents [see Eq. (1)]. This figure also presents a very good comparison between model predictions and experimental measurements for $W_{\text {eff }}$, obtained over a considerable range of work conditions.

Figures 8(b)-(c) represent, respectively, the time-average plasma potential $\bar{V}_{p}$ at position $(r=0, z=d / 2)$ and the self-bias voltage $V_{\mathrm{dc}}$, as a function of the rf applied voltage for the different frequency values considered here. An observation of these figures shows that (i) $\bar{V}_{p}$ varies very little with $f$ being an increasing function of $V_{\mathrm{rf}}$; (ii) discharge symmetry (corresponding to smaller $V_{\mathrm{dc}}$ absolute values) is favored by a reduction of both $V_{\mathrm{rf}}$ and $f$ values (see also Fig. 7). Note that a reasonable good agreement is observed between simulation results and experimental measurements for $\bar{V}_{p}$, while there is a systematic underestimation of the calculated $V_{\mathrm{dc}}$ absolute values with respect to experiment, particularly for high $V_{\mathrm{rf}}$. The latter deviations show that the model overestimates the ion current at the rf electrode (or, alternatively, that it underestimates the corresponding electron current), thus giving a strong indication that the fluid description of the rf sheath is not yet fully achieved.

\section{Influence of pressure}

This section is dedicated to analyse the influence of pressure on the main electrical features of ccrf discharges in pure hydrogen, produced in a reactor with dimensions similar to those of Sec. III C, at fixed $f=13.56 \mathrm{MHz}$ frequency, $V_{\mathrm{rf}}=350 \mathrm{~V}$ applied voltage, and for pressures in the range $p=0.5-4$ Torr.

Figure 9 represents the time-average distributions of the axial rf electric field at discharge axis, for the different pressure values considered here. From this figure one can clearly distinguish between two working regimes. For $p \lesssim 3$ Torr (at values of frequency and applied voltage adopted here), an increase in pressure yields an enlargement of the very low electric field region at discharge center, causing a simultaneous compression of discharge sheaths. The enhancement of the rf electric field within the sheaths ensures the control 
of these increasingly thinner charge separation regions. For $p \gtrsim 3$ Torr, the limitation of particle losses due to an increase in pressure allows a reduction of the electric field intensity within discharge sheaths, without compromising discharge maintenance.

Note that similar modifications upon the electric field distribution are obtained either by increasing $p$ in the low pressure regime, or by increasing $f$ at fixed pressure and applied voltage (see Sec. IIIC), as both variations lead to a limitation in the electron displacement associated to an increase of the sheath electric fields.

The above features can be related to the plot of the coupled electrical power as a function of pressure, at fixed $V_{\mathrm{rf}}=350 \mathrm{~V}$ and for different operation frequencies, which is represented in Fig. 10. As one could anticipate from the analysis of rf field changes with pressure (see Fig. 9), the variation of $W_{\text {eff }}$ with $p$ exhibits a maximum value, located around 3 Torr for $f=13.56 \mathrm{MHz}$. Moreover, this maximum shifts towards smaller $p$ values when $f$ increases, as the electron displacement limitation is favored by the combined effect of both frequency and pressure increase.

\section{E. Influence of reactor dimensions}

The important role of geometry in plasma assisted processes justifies the interest in investigate the scaling of plasma parameters with reactor dimensions. We analyse here the variation of the plasma potential, the rf electric field, the electron density and the electron mean energy with changes in the reactor radius and its interelectrode distance, whose standard dimensions are $R=6.4 \mathrm{~cm}$ and $d=3.2 \mathrm{~cm}$, respectively.

We begin the study by examining the influence of the reactor radius on a hydrogen ccrf discharge operating at $f=13.56 \mathrm{MHz}, p=0.5$ Torr and $V_{\mathrm{rf}}=200 \mathrm{~V}$, adopting a constant interelectrode distance $d=3.2 \mathrm{~cm}$ and four alternative $R$ values: $1.6 \mathrm{~cm}, 3.2 \mathrm{~cm}, 6.4 \mathrm{~cm}$ and $12.8 \mathrm{~cm}$.

Figures 11(a)-(b) plot, respectively, the time-average distributions of the plasma potential and the axial rf electric field at discharge axis, for the different values of $R$ adopted here. As expected, results show that the discharge symmetry is strongly favored by the increase of the reactor radius, as in this case the ratio of the grounded area (the surface of the grounded electrode plus that of the lateral grid) to the rf electrode area approaches unity. The magnitude of the potential drop between the plasma and the electrodes is a crucial 
parameter in deposition/etching systems, because it controls the flux of charged particles through the sheath in front of the wafer. With this respect, Fig. 11(a) shows that the discharge symmetrization, following an increase in the reactor radius, involves a simultaneous increase of the bulk potential and decrease of the self-bias voltage. In fact, between $R=$ $1.6 \mathrm{~cm}$ and $R=12.8 \mathrm{~cm}$, the absolute value of $V_{\mathrm{dc}}$ is reduced by $\sim 60 \mathrm{~V}$ (leading to a decrease of the potential drop across the rf sheath by a factor 1.3), while the potential drop across the grounded sheath increases by a factor 2. Note that the ion current is enhanced when $R$ increases (as a direct consequence of the larger area over which the ions are collected), which justifies the less negative values of the self-bias voltage. This statement can be confirmed by analysing Fig. 11(b), where a reduction of the electric field intensity is observed near the driven electrode, as $R$ increases. Note, conversely, that the electric field intensity near the grounded sheath increases with $R$, thus meaning that the ions will receive more energy when crossing that sheath.

The effective electrical power coupled to the plasma $W_{\text {eff }}$ also increases with $R$, once again due to the enlargement of the area over which the charged particles are collected. Simulations predict a variation of $W_{\text {eff }}$ from $0.18 \mathrm{~W}$ at $R=1.6 \mathrm{~cm}$ to $20.4 \mathrm{~W}$ at $R=12.8 \mathrm{~cm}$ ( $W_{\text {eff }}=5.2 \mathrm{~W}$ for standard $6.4 \mathrm{~cm}$ radius).

The impact on the electron density of reactor radius variations is analysed in Figs. 12(a)(c). The latter show the spatial contour plots of the time-average electron density, for the following reactor radii: $3.2 \mathrm{~cm}, 6.4 \mathrm{~cm}$ and $12.8 \mathrm{~cm}$. An observation of these figures reveals that: (i) the maximum electron density value is little affected by changes in $R$, remaining at $\sim 1.2 \times 10^{9} \mathrm{~cm}^{-3}$ for all situations analysed here; (ii) the maximum electron density approaches the discharge axis as $R$ decreases; (iii) the discharge symmetrization, which occurs when $R$ increases, leads to electron density profiles whose maximum is located at half distance between electrodes.

The latter result can also be confirmed by analysing Fig. 13(a), which plots the axial profile of the time-average electron density $\bar{n}_{e}$, for the different values of $R$ adopted here. At small radius, the electron density presents a steeper profile near the rf electrode, due to the very different confinement electric fields existing within the rf and grounded sheaths. As $R$ increases, results become similar to what one could expect from a one-dimensional simulation, with no self-bias voltage development and a more symmetric electron density profile between electrodes. As $R$ decreases, the small increase of the electron density is 
related to an enhancement of the electron mean energy in the $\mathrm{rf}$ sheath region. This can be observed in Fig. 13(b) which plots the axial profile of the time-average electron mean energy $\bar{\varepsilon}$, for the different values of $R$ adopted here. The variations of the mean energy intensity follow that of the average electric field $\bar{E}_{z}$ in sheath regions [see Fig. 11(b)], showing an increase of $\bar{\varepsilon}$ (hence of ionization mechanisms) near the rf electrode, and a decrease near the grounded electrode, when $R$ is reduced. Note that the discharge symmetrization leads to comparable values of $\bar{\varepsilon}$ in both sheaths, as $R$ increases.

We now examine the influence of the interelectrode distance on the same hydrogen ccrf discharge, adopting a constant reactor radius $R=6.4 \mathrm{~cm}$ and three alternative $d$ values: $2.4 \mathrm{~cm}, 3.2 \mathrm{~cm}$ and $6.4 \mathrm{~cm}$.

Figures 14(a)-(b) present, respectively, the time-average distributions of plasma potential and axial rf electric field at discharge axis, for the different values of $d$ adopted here. Results show that an increase of the interelectrode distance has three main consequences: (i) the self-bias voltage becomes more negative (see the potential value at $z=0$ ), thus indicating that the discharge is more asymmetric (the absolute value of $V_{\mathrm{dc}}$ increases $18 \%$ when $d$ is multiplied by 2); (ii) the potential at plasma bulk tends to smaller values (when $d$ changes from $2.4 \mathrm{~cm}$ to $6.4 \mathrm{~cm}$, the bulk potential decreases $14 \mathrm{~V}$ ); (iii) the potential drop across the grounded sheath is considerably reduced. Once again, these results can be understood by noting that discharge asymmetry is enhanced when $d$ increases, leading to a larger grounded grid surface. The more negative $V_{\mathrm{dc}}$ values so produced force the rf potential to shift negative during most of the rf cycle, thus yielding a reduction of the bulk potential as an overall effect. Note finally that an increase in $R$ or in $d$ is responsible for totally opposite effects in terms of the potential drop across the sheaths (see Figs. 11 and 14), as discharge symmetrization (tending to balance both potential drops) is favored by large reactor radii and small interelectrode distances.

Another important consequence of a large separation between electrodes is the increase of the effective electrical power coupled to the plasma: $W_{\text {eff }}$ increases around $30 \%$, from $5.2 \mathrm{~W}$ to $6.9 \mathrm{~W}$, when $d$ varies between $3.2 \mathrm{~cm}$ and $6.4 \mathrm{~cm}$. Although larger values of $d$ and $R$ both lead to an increase of $W_{\text {eff }}$, the physical reasons justifying these variations are quite different. In the first case, the power enhancement is due to a stronger electric field near the rf electrode [see Fig. 14(b)], while in the second case is explained by a larger collection surface for the discharge current. 
The impact on the electron density of interelectrode distance variations is analysed in Fig. 15(a), which plots the axial profile of the time-average electron density $\bar{n}_{e}$, for the different $d$ values adopted here. An observation of this figure reveals that: (i) the maximum electron density value is little affected by changes in $d$, increasing from $7 \times 10^{8} \mathrm{~cm}^{-3}$ at $d=2.4 \mathrm{~cm}$ to $1.1 \times 10^{9} \mathrm{~cm}^{-3}$ at $d=6.4 \mathrm{~cm}$; (ii) at small $d$ values the maximum electron density is symmetrically located between electrodes, but it deviates towards the rf electrode in asymmetric discharges produced at larger interelectrode distances; (iii) the density profile near the rf electrode becomes steeper at large $d$ values.

The above results can be correlated with those of Figs. 15(b)-(c), representing the axial profiles of the time-average electron mean energy $\bar{\varepsilon}$ and energy density $\overline{n_{e} \varepsilon}$, for the same $d$ values adopted before. Figure 15(b) shows an overall reduction of $\bar{\varepsilon}$ across the discharge when $d$ increases: the bulk mean energy decreases by a factor two, when $d$ varies from $2.4 \mathrm{~cm}$ to $6.4 \mathrm{~cm}$, and simultaneously the differences between $\bar{\varepsilon}$ calculated near $\mathrm{rf}$ and grounded electrodes are intensified. It is interesting to observe that, contrarily to Fig. 13(b), the electron mean energy does not follow the path of the rf sheath electric field, that slowly increases with $d$ [see Fig. 14(b)]. Instead, this behavior is exhibited by the electron energy density [see Fig. 15(c)], whose variations are mainly controlled by $\bar{n}_{e}$. Figure $15(\mathrm{c})$ shows another interesting feature: the energy density presents a small ripple in the transition regions between bulk and sheaths. These slope variations are induced by the very steep decrease of the electron mean energy in these regions (where ionization events occur mainly), combined with non-locality effects which place the maxima of $\bar{n}_{e}$ and $\bar{\varepsilon}$ at different locations.

Figure 16 represents the spatial contour plots of the time-average electron density, for the interelectrode distances considered here. As expected, one observes that, as $d$ increases, the region of higher electron density expands towards the discharge axis, also approaching the rf electrode as a result of discharge asymmetry enhancement.

\section{F. Dissociation rate}

In CVD plasma reactors, it seems clear that the presence of hydrogen atoms tends to favor the microcristallin structure of the deposited films. In an attempt to define the operating conditions that enhance $\mathrm{H}$ production, the space-time average of the $\mathrm{H}_{2}$ dissociation rate by electron impact $\left\langle\bar{S}_{d}\right\rangle$ is plotted as a function of pressure, in Fig. $17($ a) for $f=13.56 \mathrm{MHz}$ at 
different $V_{\text {rf }}$ values, and in Fig. $17(\mathrm{~b})$ for $V_{\text {rf }}=350 \mathrm{~V}$ at different frequencies. An observation of these figures reveals that the production rate of hydrogen atoms increases with both the applied voltage and frequency, presenting a maximum for a pressure value that increases with $V_{\text {rf }}$ and decreases with $f$. As expected, the latter variations closely follow those observed for the coupled electrical power, with changes in the applied voltage, pressure and frequency [see Figs. 8(a) and 10].

The hydrogen dissociation rate increases with both $V_{\mathrm{rf}}$ and $f$ due to the combined enhancement of the rf electric field [see Figs. 7 and 8(b)] and the plasma electron density (see Fig. 6). At low pressures, $\left\langle\bar{S}_{d}\right\rangle$ increases with $p$ not only because of the direct increase in gas density, but also due to the increase of the sheath electric fields (see Sec. III C). At high pressures, the decrease of $\left\langle\bar{S}_{d}\right\rangle$ as $p$ increases is associated to the reduction of the electric field intensity within discharge sheaths, as a consequence of particle loss limitations (see Fig. 9).

The time-average 2D profile and the space-time variation (during one rf period at discharge axis) of the hydrogen dissociation rate $S_{d}$ are somewhat similar to those for the hydrogen ionization rate [see Figs. $3(\mathrm{a})-(\mathrm{b})]$. The time-average $2 \mathrm{D}$ profile $\bar{S}_{d}(r, z)$ presents a maximum value of about $10^{16} \mathrm{~cm}^{-3} \mathrm{~s}^{-1}$, located at $z \simeq 1 \mathrm{~cm}$ and $r \simeq 5.0 \mathrm{~cm}$, being approximately constant and equal to $6 \times 10^{15} \mathrm{~cm}^{-3} \mathrm{~s}^{-1}$ within a radial distance of $4 \mathrm{~cm}$ from discharge axis. The latter observation can be important when analysing the operation of plasma reactors for deposition, since it provides an indication of the region where film uniformity can be expected. In what concerns space-time variation, the highest peak of $S_{d}(0, z, t)$ has an intensity of about $1.6 \times 10^{16} \mathrm{~cm}^{-3} \mathrm{~s}^{-1}$ and it occurs during the rf sheath expansion at $z \simeq 1 \mathrm{~cm}$ and $t \simeq 27 \mathrm{~ns}$.

\section{SIMILARITY LAW}

This section presents a systematic study of the rf field variations in hydrogen ccrf discharges, with changes in applied voltage, pressure and geometric dimensions, at fixed $f=13.56 \mathrm{MHz}$ operating frequency. The aim of this study is to show that these variations can be described using an universal similarity law, if an adequate normalization is adopted when plotting the rf electric field as a function of pressure.

Similarity laws can be of valuable interest in the definition of working regimes for a given 
type of discharge. The problem has been extensively studied, both theoretically and experimentally, in direct-current ${ }^{24-28}$ and microwave ${ }^{29-34}$ low pressures discharges, because in general the description of these systems allows important simplifications concerning geometry and time evolution. Unfortunately, this is not the case with rf discharges, where a detailed space-time analysis is required in order to achieve correct predictions. The problem can be circumvented by representing each discharge condition through a single value of the rf electric field $\left\langle\bar{E}_{\mathrm{rms}}\right\rangle$, obtained by calculating the root-mean-square (rms) in time, and the average value in space, of the total electric field space-time distribution $(\langle\rangle$ refers to the spatial average).

The present simulations cover a large domain of pressures (0.25 - 8.0 Torr), for different applied voltages $(217 \mathrm{~V}, 350 \mathrm{~V}$ and $450 \mathrm{~V})$, and interelectrode distances $(1.6 \mathrm{~cm}, 3.2 \mathrm{~cm}$ and $6.4 \mathrm{~cm}$ ). In a first set of simulations, $p$ was varied at different $V_{\mathrm{rf}}$ values by keeping a constant interelectrode distance $d=3.2 \mathrm{~cm}$. In a second set of simulations $p$ was varied at different $d$ values by keeping a constant rf applied voltage $V_{\mathrm{rf}}=350 \mathrm{~V}$.

Figures 18(a)-(b) plot the space-average, rms electric field as a function of pressure, for the different values of applied voltages and interelectrode distances adopted here. From these figures one observes that the variation of $\left\langle\bar{E}_{\mathrm{rms}}\right\rangle$ with $p$ exhibits a maximum for fixed values of either $V_{\mathrm{rf}}$ or $d$. At low pressures, the enlargement of the very low electric field region at discharge center (due to a compression of sheaths) demands an increase of $\left\langle\bar{E}_{\mathrm{rms}}\right\rangle$ with $p$, in order to ensure discharge maintenance. At high pressures, charged particle losses are strongly limited by diffusion, and thus the discharge operating point can be obtained for increasingly smaller $\left\langle\bar{E}_{\text {rms }}\right\rangle$ values, as $p$ increases (see also Sec. III D and Figs. 9-10). Note that a reduction in losses due to transport restrictions also accounts for the decrease of $\left\langle\bar{E}_{\mathrm{rms}}\right\rangle$ with the increase of $d$ (at constant $V_{\mathrm{rf}}$ and $p$ ) [see Fig. 18(b)]. Note finally that, as expected, when $V_{\text {rf }}$ increases at constant $d$ the values of $\left\langle\bar{E}_{\mathrm{rms}}\right\rangle$ are strongly enhanced, with the maximum of the field being shifted towards higher pressures [see Fig. 18(a)].

As stated before, the main purpose here is the introduction of adequate variables in order to align the different sets of points in Figs. 18(a)-(b), and obtain an universal similarity curve. The interest with this representation is that it allows a univocal definition of a reactor working point, for given operating conditions: rf voltage, gas pressure and interelectrode distance. The strategy to be follow in defining a similarity curve for rf discharges proceeds in two steps. 
The first step starts by recognizing that the universal characterization of rf discharges must involve the same set of parameters used to describe their operation features. This set corresponds to the reduced quantities $\bar{E}_{\mathrm{rms}, i} / N(i=r, z), N R$ and $N d$, as can be deduced from a direct inspection of the charged particle and energy, time-averaged transport equations. ${ }^{2}$ Because here we are working at constant reactor radius, and we have chosen to represent the rf electric field by its space-average rms value, our base start parameter set can be reduced to $\left\langle\bar{E}_{\mathrm{rms}} / N\right\rangle$ and $N d$. The latter quantities are plotted against each other in Fig. 19, for the different values of applied voltages $V_{\mathrm{rf}}$ and interelectrode distances $d$ considered here.

The second and final step in our strategy involves the introduction of two similarity parameters, $E^{\star} / N$ and $N \Lambda$, to be obtained from an adequate normalization of $\left\langle\bar{E}_{\mathrm{rms}} / N\right\rangle$ and $N d$, respectively. In particular, the definition of these similarity parameters must ensure that the different sets of points in Fig. 19 collapse onto the ones corresponding to some reference conditions, if this figure is re-plotted using $E^{\star} / N$ and $N \Lambda$ as new coordinates. In what follows, the operation conditions corresponding to $V_{\mathrm{rf}}=350 \mathrm{~V}$ and $d=3.2 \mathrm{~cm}$ will be taken as reference conditions.

For a fixed value of $d=d_{\text {ref }}=3.2 \mathrm{~cm}$, the variations of $\left\langle\bar{E}_{\mathrm{rms}}\right\rangle / N$ with pressure are accompanied by strong and unequal variations of $W_{\text {eff }}$ (see Fig. 10). Since the coupled electrical power is proportional to the square of the electric field, the similarity parameter representing the space-average, rms electric field, normalized to power $1 \mathrm{~W}$, is defined as

$$
\frac{E^{\star}}{N} \equiv \frac{\left\langle\bar{E}_{\mathrm{rms}}\right\rangle / N}{W_{\mathrm{eff}}^{1 / 2}}
$$

The second similarity parameter, $N \Lambda$, allows a representation that includes different interelectrode distances. The quantity $\Lambda$ corresponds to an effective length defined as

$$
\Lambda \equiv d \frac{d_{\mathrm{ref}} / l_{\mathrm{ref}}}{d / l}
$$

where $l$ represents the axial thickness of the $\mathrm{rf}$ sheath, $l_{\text {ref }}$ being the corresponding thickness obtained for $d=d_{\text {ref }}$. The values of the $\operatorname{rf}$ sheath thickness $l$ are estimated as the distance between the rf electrode and the axial position where the time-average electric field $\bar{E}_{z}$ goes to zero [see, for example, Figs. 11(b) or 14(b)]. With this definition, $\Lambda$ can be seen as a corrected value of the reference interelectrode distance, according to the relative variation 
of the rf sheath thickness. In fact, equation (4) can be rewritten as

$$
\Lambda=d_{\mathrm{ref}} \frac{l}{l_{\mathrm{ref}}}
$$

thus showing that the similarity parameter $\Lambda$ acts as follows

$$
\begin{aligned}
& \Lambda<d, \quad \text { for } d>d_{\text {ref }} \Rightarrow l<l_{\text {ref }} \\
& \Lambda \geq d, \quad \text { for } d \leq d_{\text {ref }} \Rightarrow l \geq l_{\text {ref }} .
\end{aligned}
$$

Figure 20 represents the new plot of $E^{\star} / N$ as a function of $N \Lambda$, for the various $V_{\text {rf }}$ and $d$ values considered in Fig. 19. The figure confirms that the similarity parameters $E^{\star} / N$ and $N \Lambda$ were adequately define, as they allow to group very different discharge conditions under the same characteristic curve.

The universal feature of this representation can be randomly tested. For arbitrary $p=$ 0.5 Torr, $V_{\text {rf }}=280 \mathrm{~V}$ and $d=d_{\text {ref }}$ operating conditions, simulation results yield $\left\langle\bar{E}_{\mathrm{rms}}\right\rangle / N=$ $115.67 \times 10^{-16} \mathrm{Vcm}^{2}$ and $W_{\text {eff }}=9.78 \mathrm{~W}$, corresponding to similarity parameters

$$
\begin{aligned}
N \Lambda & =5.15 \times 10^{16} \mathrm{~cm}^{-2} \\
\left(\left\langle\bar{E}_{\mathrm{rms}}\right\rangle / N\right)\left(W_{\mathrm{eff}}\right)^{-1 / 2} & =36.99 \times 10^{-16} \mathrm{Vcm}^{2},
\end{aligned}
$$

which fairly fit the similarity curve of Fig. 20. For arbitrary $p=0.5$ Torr, $V_{\mathrm{rf}}=200 \mathrm{~V}$ and $d=$ $2.4 \mathrm{~cm}$ operating conditions, simulation results yield $\left\langle\bar{E}_{\mathrm{rms}}\right\rangle / N=89.76 \times 10^{-16} \mathrm{Vcm}^{2}, W_{\mathrm{eff}}=$ $4.17 \mathrm{~W}$ and $\Lambda=3.36 \mathrm{~cm}$, corresponding to similarity parameters

$$
\begin{aligned}
N \Lambda & =5.4 \times 10^{16} \mathrm{~cm}^{-2} \\
\left(\left\langle\bar{E}_{\mathrm{rms}}\right\rangle / N\right)\left(W_{\mathrm{eff}}\right)^{-\frac{1}{2}} & =43.95 \times 10^{-16} \mathrm{Vcm}^{2},
\end{aligned}
$$

which again fit the similarity curve of Fig. 20 quite well. The latter two operation points are represented by the closed circles in this figure.

The similarity law pictured in Fig. 20 can be extremely useful to define the working points of ccrf discharges in hydrogen, as well as to obtain their space-time average electric field for given coupled power, pressure, sheath thickness, and interelectrode distance. Note that the similarity law defined in this section is for a fixed operating frequency of $13.56 \mathrm{MHz}$. In principle, the results obtained here can be generalized to discharges operating at different frequencies, similarly to what was previously done in microwave discharges. ${ }^{29}$ 


\section{SUMMARY}

This paper has presented a systematic characterization of pure hydrogen ccrf discharges, produced in a cylindrical parallel plate reactor, similar to the GEC reference Cell. A twodimensional, time-dependent fluid model was used to describe the production, transport and destruction of electrons and positive ions $\mathrm{H}^{+}, \mathrm{H}_{2}^{+}$, and $\mathrm{H}_{3}^{+}$, at different frequencies $(13.56-60 \mathrm{MHz})$, pressures $(0.2-8$ Torr $)$, rf applied voltages $(50-450 \mathrm{~V})$ and geometric dimensions (1.6 - $12.8 \mathrm{~cm}$ radii and $1.6-6.4 \mathrm{~cm}$ interelectrode distances).

Simulations show that $\mathrm{H}_{3}^{+}$is the dominant ion species, its maximum density being more than one order of magnitude higher than the mean densities of either $\mathrm{H}_{2}^{+}$or $\mathrm{H}^{+}$. The hydrogen ionization rate has a time-average profile with two maxima located at the bulksheath interface, near the reactor corners, and presents a space-time variation (at discharge axis) showing the development of double ionization structures in front of the electrodes, at instants associated with the occurrence of the maximum expansion and contraction phases of discharge sheaths.

The time evolution of the axial electric field reveals the formation of a double sheath at the early beginning of each time period (before the rf sheath starts its expansion), in order to further limit the entrance of electrons in sheath region. The development of this double sheath leads to a field inversion phenomenon, characterized by a positive $E_{z}$ region near the rf electrode (due to the electron accumulation at sheath border), followed by a negative value region for this field. In some extreme conditions (yielding an enhanced ion flow towards the rf electrode), it is also possible to observe a field reversal situation for a very short time interval at the end of the $\mathrm{rf}$ period, during which the positive $E_{z}$ region enlarges and the sheath field becomes oriented towards the discharge bulk.

The dependencies on pressure and frequency of the time-average electric field distribution were analysed and related to the electron displacement within space-charge sheaths. It is observed that an increase in the excitation frequency has the following major consequences: (i) a reduction of sheath thickness and a discharge asymmetrization; (ii) an enhancement of the ionization rate leading to higher electron densities; (iii) an increase of the electrical power coupled to the plasma, due to an enhancement of the total current flowing to the driven electrode. Furthermore, a similar enhancement of the sheath electric fields is obtained either by increasing $p$ in the low pressure regime $(\lesssim 3$ Torr, for the values of frequency and 
applied voltage adopted here), or by increasing $f$ at fixed pressure and applied voltage, as both variations lead to a limitation of the electron displacement associated to a sheath compression. At higher pressures, however, the limitation of particle losses as $p$ increases allows a reduction of the electric field intensity within discharge sheaths, which leads to a discharge symmetrization. These features are retrieved when analysing the variations, with changes in discharge operating conditions, of the coupled electrical power or the space-time averaged hydrogen dissociation rate by electron impact. In fact, the plots of both $W_{\text {eff }}$ and $\left\langle\bar{S}_{d}\right\rangle$, as a function of pressure, exhibit a maximum value that shifts towards smaller $p$ values when $f$ increases, and towards higher $p$ values when $V_{\text {rf }}$ increases.

The influence of reactor dimensions in the spatial profiles of the plasma potential, the rf electric field, the electron density and the electron mean energy were also investigated. Results confirm that an increase of the bulk potential and a discharge symmetrization are favored by large reactor radii and small interelectrode distances, as in these cases the ratio of the grounded area to the rf electrode area approaches unity. However, the electrical power coupled increases with both $R$ and $d$. In the first case, the power enhancement is due to a stronger electric field near the rf electrode, while in the second case is explained by a larger collection surface for the discharge current.

An investigation of the space-time averaged rf electric field variations, with changes in the applied voltage, pressure, and geometric dimensions is carried out. These variations are shown to follow an universal similarity curve, if the plot of the rf electric field as a function of pressure uses the following similarity parameters: $E^{\star} / N \equiv\left(\left\langle\bar{E}_{\mathrm{rms}}\right\rangle / N\right)\left(W_{\text {eff }}\right)^{-1 / 2}$ (rms accounts for root-mean-square in time; 〈〉 refers to spatial average) and $N \Lambda \equiv$ $N d\left(d_{\text {ref }} / l_{\text {ref }}\right)(d / l)^{-1}$, where $l$ represents the axial thickness of the $\mathrm{rf}$ sheath, $l_{\text {ref }}$ being the corresponding thickness obtained for some reference value of the interelectrode distance $\left(d=d_{\text {ref }}\right)$. This innovative representation of rf discharges allows a univocal definition of a reactor working point, for given operating conditions, and can be used to obtain its space-time average electric field for given coupled power, pressure, sheath thickness, and interelectrode distance.

A general good agreement was found between model results and experimental measurements for the coupled electrical power, the plasma potential and the self-bias potential, at various frequencies and rf applied voltages. However, the values of the calculated electron densities are a factor $3-7$ bellow the measured ones, and this difference is aggravated at 
both low applied voltages and frequencies. The observed disagreement is most probably associated to a combination of factors: the still incomplete fluid description of rf space-charge sheaths (for example, due to the absence of the non-linear inertia term in the electron flux equation), and the simplified hydrogen kinetics considered here. Work is in progress to overcome these model limitations.

\section{Acknowledgments}

A. Salabas acknowledges the Portuguese Ministry of Science and University-Teaching for his fellowship PRAXIS XXI (BD/15716/98) at Centro de Física dos Plasmas, Instituto Superior Técnico, Lisbon.

L. Marques acknowledges the financial support of the bureau GRICES and the French Embassy, for his fellowship at Laboratoire de Physique des Gaz et des Plasmas, Université Paris-Sud, France, in the framework of the Scientific and Technical Luso-French Cooperation Program, Project 539-B4. He also acknowledges the Portuguese Ministry of Science and University-Teaching for his fellowship SFRH/BD/5012/2001 at Centro de Física dos Plasmas, Instituto Superior Técnico, Lisbon. 
1 P. Kae-Nune, J. Perrin, J. Guillon, and J. Jolly, Jpn. J. Appl. Phys. 33, 4303 (1994); Plasma Sources Sci. Technol. 4, 250 (1995).

2 A. Salabas, G. Gousset, and L. L. Alves, Plasma Sources Sci. Technol. 11, 448 (2002).

3 A. Salabas, Ph.D. thesis, Lisbon Technical University, 2003.

4 T. Simko, V. Martisovits, J. Bretagne, and G. Gousset, Phys. Rev. E 56, 5908 (1997).

5 H. Tawara, Y. Itikawa, H. Nishimura, and M. Yoshino, J. Phys. Chem. Ref. Data 19, 617 (1990).

6 J. Loureiro and C. M. Ferreira, J. Phys. D 22, 1680 (1989).

7 D. Rapp and P. Englander-Golden, J. Chem. Phys. 43, 1464 (1965).

8 M. J. Kushner, J. Appl. Phys. 63, 2532 (1988).

9 J. Perrin, O. Leroy, and M. C. Bordage, Contrib. Plasma Phys. 36, 3 (1996).

10 C. F. Chan, "Reaction Cross-Sections and Rate Coefficients Related to the Production of Positive Ions", Lawrence Berkeley Lab. Report No. LBID-632, 1983.

11 L. P. Theard and W. T. Huntress, Jr., J. Chem. Phys. 60, 2840 (1974).

12 L. Marques, A. Salabas, G. Gousset, J. Jolly, and L. L. Alves, in Proceedings of the $16^{\text {th }}$ European Conference on Atomic \& Molecular Physics of Ionized Gases, and $5^{\text {th }}$ International Conference on Reactive Plasmas, edited by N. Sadeghi and H. Sugai (Grenoble, France, 2002), pp. 69-70.

13 J. Bretagne, G. Gousset, and T. Simko, J. Phys. D 27, 1866 (1994).

14 A. V. Phelps, J. Chem. Phys. Ref. Data 19, 653 (1990).

15 E. W. McDaniel and E. A. Mason, The Mobility and Diffusion of Ions in Gases (Wiley, New York, 1973).

16 A. L. Ward, J. Appl. Phys. 33, 2789 (1962).

17 O. Leroy, P. Stratil, J. Perrin, J. Jolly, and P. Bellenguer, J. Phys. D 28, 500 (1995).

18 W. G. Graham and C. M. O. Mahoney, "Experimental studies of rf sheath" in Electron Kinetics and Applications of Glow Discharges, edited by U. Kortshagen and L. Tsendin (NATO ASI Series B: Physics, Plenum, New York, 1998), Vol. 367, pp. 503-510.

19 M. Heintze, R. Zedlitz, and G. H. Bauer, J. Phys. D 26, 1781 (1993).

20 S. Oda, Plasma Sources Sci. Technol. 2, 26 (1993).

21 T. Kitamura, N. Nakano, T. Makabe, and Y. Yamaguchi, Plasma Sources Sci. Technol. 2, 40 (1993). 
22 V. Vahedi, C. K. Birdsall, M. A. Lieberman, G. DiPeso, and T. D. Rognlien, Phys. Fluids B 5, 2719 (1993).

23 M. Yan and W. J. Goedheer, Plasma Sources Sci. Technol. 8, 349 (1999).

24 S. A. Self and H. N Ewald, Phys. Fluids 9, 2486 (1966).

25 J. H. Ingold, Phys. Fluids 15, 75 (1972).

26 C. M. Ferreira, J. Loureiro, and A. Ricard, J. Appl. Phys. 57, 82 (1985).

27 E. Kawamura and J. H. Ingold, J. Phys. D 34, 3150 (2001).

28 L. L. Alves, G. Gousset, and S. Valée, IEEE Trans. Plasma Sci., to be published (2003).

29 C. M. Ferreira and J. Loureiro, J. Phys. D 17, 1175 (1984).

30 C. M. Ferreira and M. Moisan, Physica Scripta 38, 382 (1988).

31 C. Boisse-Laporte, A. Granier, E. Dervisevic, P. Leprince, and J. Marec, J. Phys. D 20, 197 (1987); S. Daviaud, C. Boisse-Laporte, P. Leprince, and J. Marec, ibid. 22, 770 (1989); A. Granier, S. Pasquiers, C. Boisse-Laporte, R. Darchicourt, P. Leprince, and J. Marec, ibid. 22, $1487(1989)$.

32 M. Moisan, C. Barbeau, R. Claude, C. M. Ferreira, J. Margot, J. Paraszczak, A. B. Sá, G. Sauvé, and M. R. Wertheimer, J. Vac. Sci. Technol. B 9, 8 (1991).

33 C. M. Ferreira, L. L. Alves, M. Pinheiro, and A. B. Sá, IEEE Trans. Plasma Sci. 19, 229 (1991).

34 P. A. Sá, J. Loureiro, and C. M. Ferreira, J. Phys. D 25, 960 (1992). 
TABLE I: Charged particle production and destruction mechanisms in hydrogen.

\begin{tabular}{lllll}
\hline \hline & Reaction & & $\begin{array}{c}\text { Rate coefficient }^{a} \\
\left(\mathbf{c m}^{3} \mathbf{~ s}^{-1}\right)\end{array}$ & References \\
\hline $\mathrm{e}+\mathrm{H}_{2}$ & $\stackrel{0.93^{b}}{\longrightarrow}$ & $2 \mathrm{e}+\mathrm{H}_{2}^{+}$ & $\mathrm{EBE}$ & $5-7$ \\
& $\stackrel{0.07^{b}}{\longrightarrow}$ & $2 \mathrm{e}+\mathrm{H}^{+}+\mathrm{H}$ & $\mathrm{EBE}$ & $5-7$ \\
$\mathrm{e}+\mathrm{H}_{3}^{+}$ & $\longrightarrow$ & $\mathrm{H}_{2}+\mathrm{H}$ & $9.75 \times 10^{-8} T_{e}^{-0.5}$ & $8-10$ \\
$\mathrm{e}+\mathrm{H}_{2}^{+}$ & $\longrightarrow$ & $\mathrm{H}+\mathrm{H}$ & $5.66 \times 10^{-8} T_{e}^{-0.5}$ & $8-10$ \\
$\mathrm{e}+\mathrm{H}^{+}$ & $\longrightarrow$ & $\mathrm{H}$ & $2.62 \times 10^{-13} T_{e}^{-0.5}$ & $8-10$ \\
$\mathrm{H}_{2}^{+}+\mathrm{H}_{2}$ & $\longrightarrow$ & $\mathrm{H}_{3}^{+}+\mathrm{H}$ & $2.10 \times 10^{-9}$ & 8,11 \\
\hline \hline
\end{tabular}

${ }^{a}$ The label EBE (electron Boltzmann equation) means that the rate coefficient is calculated from the corresponding electron collision cross-section, using the electron energy distribution function obtained from the solution to the homogeneous and stationary EBE. In this column $T_{e}$ is in $\mathrm{eV}$.

${ }^{b}$ Branching ratio from Refs. 4,5 . 
FIG. 1: Time-average, 2D steady-state profiles of the electron density (a) and electron mean energy (b), for a hydrogen ccrf discharge operating at $f=13.56 \mathrm{MHz}, V_{\mathrm{rf}}=200 \mathrm{~V}, p=0.5$ Torr, and reactor dimensions $R=6.4 \mathrm{~cm}$ and $d=3.2 \mathrm{~cm}$.

FIG. 2: Time-average, 2D steady-state density profiles of the following hydrogen ions: $\mathrm{H}^{+}(\mathbf{a})$, $\mathrm{H}_{2}^{+}$(b) and $\mathrm{H}_{3}^{+}$(c). Results were obtained in the same conditions of Fig. 1.

FIG. 3: Time-averaged spatial contour plot (a) and space-time variation (at $r=0$ ) during one rf cycle (b) of the hydrogen ionization rate (in $\mathrm{cm}^{-3} \mathrm{~s}^{-1}$ ), for a discharge operating in the same conditions of Fig. 1.

FIG. 4: Spatial profile (at $r=0$ ) of the axial electric field $E_{z}$, at different times within one rf period: (a) 0 (solid line); $T_{\mathrm{rf}} / 10$ (dashed); $2 T_{\mathrm{rf}} / 10$ (dotted); $3 T_{\mathrm{rf}} / 10$ (dashed-dotted); 4T $T_{\mathrm{rf}} / 10$ (dashed-dotted-dotted); (b) $5 T_{\mathrm{rf}} / 10$ (solid); $6 T_{\mathrm{rf}} / 10$ (dashed); $7 T_{\mathrm{rf}} / 10$ (dotted); $8 T_{\mathrm{rf}} / 10$ (dasheddotted); $9 T_{\text {rf }} / 10$ (dashed-dotted-dotted). Results were obtained in the same conditions of Fig. 1.

FIG. 5: Zoom over the spatial profile (at $r=0$ ) of the axial electric field $E_{z}$ at $t=T_{\mathrm{rf}} / 20$, obtained in the same conditions of Fig. 4 for the following $V_{\text {rf }}$ values: $200 \mathrm{~V}$ (solid line); $400 \mathrm{~V}$ (dashed). Notice the formation of a double sheath structure, associated to the field inversion phenomenon (see text).

FIG. 6: Time-average electron density (at $r=0$ and $z=d / 2$ ) as a function of $V_{\mathrm{rf}}$, for hydrogen ccrf discharges operating at $p=0.3$ Torr, reactor dimensions $R=6.4 \mathrm{~cm}$ and $d=3.0 \mathrm{~cm}$, and the following frequency values: $13.56 \mathrm{MHz}$ (solid curve); $27.12 \mathrm{MHz}$ (dashed); $40.68 \mathrm{MHz}$ (dotted). The points correspond to experimental measurements obtained under the same discharge conditions at frequencies 13.56 MHz (squares), 27.12 MHz (circles) and 40.68 MHz (triangles). The experimental values were divided by 3 for representation purposes.

FIG. 7: Spatial profile (at $r=0$ ) of the time-average, steady-state axial electric field $\bar{E}_{z}$, for hydrogen ccrf discharges operating at $V_{\mathrm{rf}}=100 \mathrm{~V}, p=0.3$ Torr, reactor dimensions $R=6.4 \mathrm{~cm}$ and $d=3.0 \mathrm{~cm}$, and the following frequency values: $13.56 \mathrm{MHz}$ (solid curve); $27.12 \mathrm{MHz}$ (dashed); 40.68 MHz (dotted); $60 \mathrm{MHz}$ (dashed-dotted). 
FIG. 8: Electrical parameters as a function of $V_{\mathrm{rf}}$, for discharges operating in the same conditions of Fig. 6. Effective electrical power coupled to the plasma, $W_{\text {eff }}(\mathbf{a})$; time-average, steady-state plasma potential (at $r=0$ and $z=d / 2), \bar{V}_{p}(0, d / 2)$ (b); self-bias voltage, $V_{\mathrm{dc}}(\mathbf{c})$. The points correspond to experimental measurements obtained under the same discharge conditions at frequencies 13.56 MHz (squares), 27.12 MHz (circles) and 40.68 MHz (triangles).

FIG. 9: Spatial profile (at $r=0$ ) of the time-average, steady-state axial electric field $\bar{E}_{z}$, for hydrogen ccrf discharges operating at $f=13.56 \mathrm{MHz}, V_{\mathrm{rf}}=350 \mathrm{~V}$, reactor dimensions $R=6.4 \mathrm{~cm}$ and $d=3.0 \mathrm{~cm}$, and the following pressure values: 0.5 Torr (solid curve); 1 Torr (dashed); 2 Torr (dotted); 3 Torr (dashed-dotted); 4 Torr (dashed-dotted-dotted).

FIG. 10: Effective electrical power coupled to the plasma as a function of pressure, for hydrogen ccrf discharges operating at $V_{\mathrm{rf}}=350 \mathrm{~V}$, reactor dimensions $R=6.4 \mathrm{~cm}$ and $d=3.0 \mathrm{~cm}$, and the following frequency values: $13.56 \mathrm{MHz}$ (solid curve); $27.12 \mathrm{MHz}$ (dashed); $40.68 \mathrm{MHz}$ (dotted).

FIG. 11: Spatial profile (at $r=0$ ) of the time-average, steady-state plasma potential $\bar{V}_{p}$ (a) and axial electric field $\bar{E}_{z}$ (b). Results were obtained in the same conditions of Fig. 1, for $d=3.2 \mathrm{~cm}$ and the following values of reactor radius $R: 1.6 \mathrm{~cm}$ (solid line); $3.2 \mathrm{~cm}$ (dashed), $6.4 \mathrm{~cm}$ (dotted); $12.8 \mathrm{~cm}$ (dashed-dotted).

FIG. 12: Time-averaged spatial contour plot of the steady-state electron density $\left(\right.$ in $\mathrm{cm}^{-3}$ ), for hydrogen discharges operating in conditions of Fig. 1, at $d=3.2 \mathrm{~cm}$ and the following values of

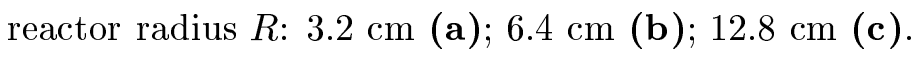

FIG. 13: Spatial profile (at $r=0$ ) of the time-average, steady-state electron density $\bar{n}_{e}$ (a) and electron mean energy $\bar{\varepsilon}(\mathbf{b})$. Results were obtained in the same conditions of Fig. 11.

FIG. 14: Spatial profile (at $r=0$ ) of the time-average, steady-state plasma potential $\bar{V}_{p}$ (a) and axial electric field $\bar{E}_{z}$ (b). Results were obtained in the same conditions of Fig. 1, for $R=6.4 \mathrm{~cm}$ and the following values of interelectrode distance $d: 2.4 \mathrm{~cm}$ (solid line); $3.2 \mathrm{~cm}$ (dashed); $6.4 \mathrm{~cm}$ (dotted). 
FIG. 15: Spatial profile (at $r=0$ ) of the time-average, steady-state electron density $\bar{n}_{e}(\mathbf{a})$, electron mean energy $\bar{\varepsilon}(\mathbf{b})$, and energy density $\overline{n_{e} \varepsilon}(\mathbf{c})$. Results were obtained in the same conditions of Fig. 14.

FIG. 16: Time-averaged spatial contour plot of the steady-state electron density $\left(\right.$ in $\mathrm{cm}^{-3}$ ), for hydrogen discharges operating in conditions of Fig. 1, at $R=6.4 \mathrm{~cm}$ and the following values of interelectrode distance $d: 2.4 \mathrm{~cm} \mathrm{(a);3.2} \mathrm{cm} \mathrm{(b);} 6.4 \mathrm{~cm} \mathrm{(c)}$.

FIG. 17: Space-time averaged dissociation rate of hydrogen by electron impact $\left\langle\bar{S}_{d}\right\rangle$, as a function of pressure. (a) Results obtained at $f=13.56 \mathrm{MHz}$, reactor dimensions $R=6.4 \mathrm{~cm}$ and $d=3.2 \mathrm{~cm}$, and the following $V_{\mathrm{rf}}$ values: $217 \mathrm{~V}$ (solid line); $350 \mathrm{~V}$ (dashed); $450 \mathrm{~V}$ (dotted). (b) Results obtained at $V_{\mathrm{rf}}=350 \mathrm{~V}$, reactor dimensions $R=6.4 \mathrm{~cm}$ and $d=3.0 \mathrm{~cm}$, and the following $f$ values: $13.56 \mathrm{MHz}$ (solid curve); 27.12 MHz (dashed); $40.68 \mathrm{MHz}$ (dotted). 
FIG. 18: Space-average, rms electric field $\left\langle\bar{E}_{\mathrm{rms}}\right\rangle$, as a function of $p$, for hydrogen ccrf discharges at $f=13.56 \mathrm{MHz}$. (a) Results for $d=3.2 \mathrm{~cm}$ and the following $V_{\mathrm{rf}}$ values: $217 \mathrm{~V}$ (squares); $350 \mathrm{~V}$ (circles); $450 \mathrm{~V}$ (triangles). (b) Results for $V_{\mathrm{rf}}=350 \mathrm{~V}$ and the following $d$ values: $1.6 \mathrm{~cm}$ (squares); $3.2 \mathrm{~cm}$ (circles); $6.4 \mathrm{~cm}$ (triangles).

FIG. 19: Space-average, rms reduced electric field $\left\langle\bar{E}_{\mathrm{rms}} / N\right\rangle$, as a function of $N d$, for hydrogen ccrf discharges at $f=13.56 \mathrm{MHz}$ and the following rf applied voltages and interelectrode distances: $V_{\mathrm{rf}}=217 \mathrm{~V}$ and $d=d_{\text {ref }}=3.2 \mathrm{~cm}$ (squares); $V_{\mathrm{rf}}=350 \mathrm{~V}$ and $d=d_{\text {ref }}$ (circles); $V_{\mathrm{rf}}=450 \mathrm{~V}$ and $d=d_{\text {ref }}$ (up triangles); $V_{\mathrm{rf}}=350 \mathrm{~V}$ and $d=d_{\text {ref }} / 2$ (down triangles); $V_{\mathrm{rf}}=350 \mathrm{~V}$ and $d=2 d_{\text {ref }}$ (diamonds).

FIG. 20: Similarity curve of $E^{\star} / N \equiv\left(\left\langle\bar{E}_{\mathrm{rms}}\right\rangle / N\right)\left(W_{\mathrm{eff}}\right)^{-1 / 2}$ vs. $N \Lambda\left[\Lambda \equiv d\left(d_{\mathrm{ref}} / l_{\mathrm{ref}}\right)(d / l)^{-1}\right]$, obtained for hydrogen ccrf discharges operating at $f=13.56 \mathrm{MHz}$ and the rf applied voltages and interelectrode distances of Fig. 19. The closed circles are for two arbitrarily defined operation points, used to test this representation (see text). 

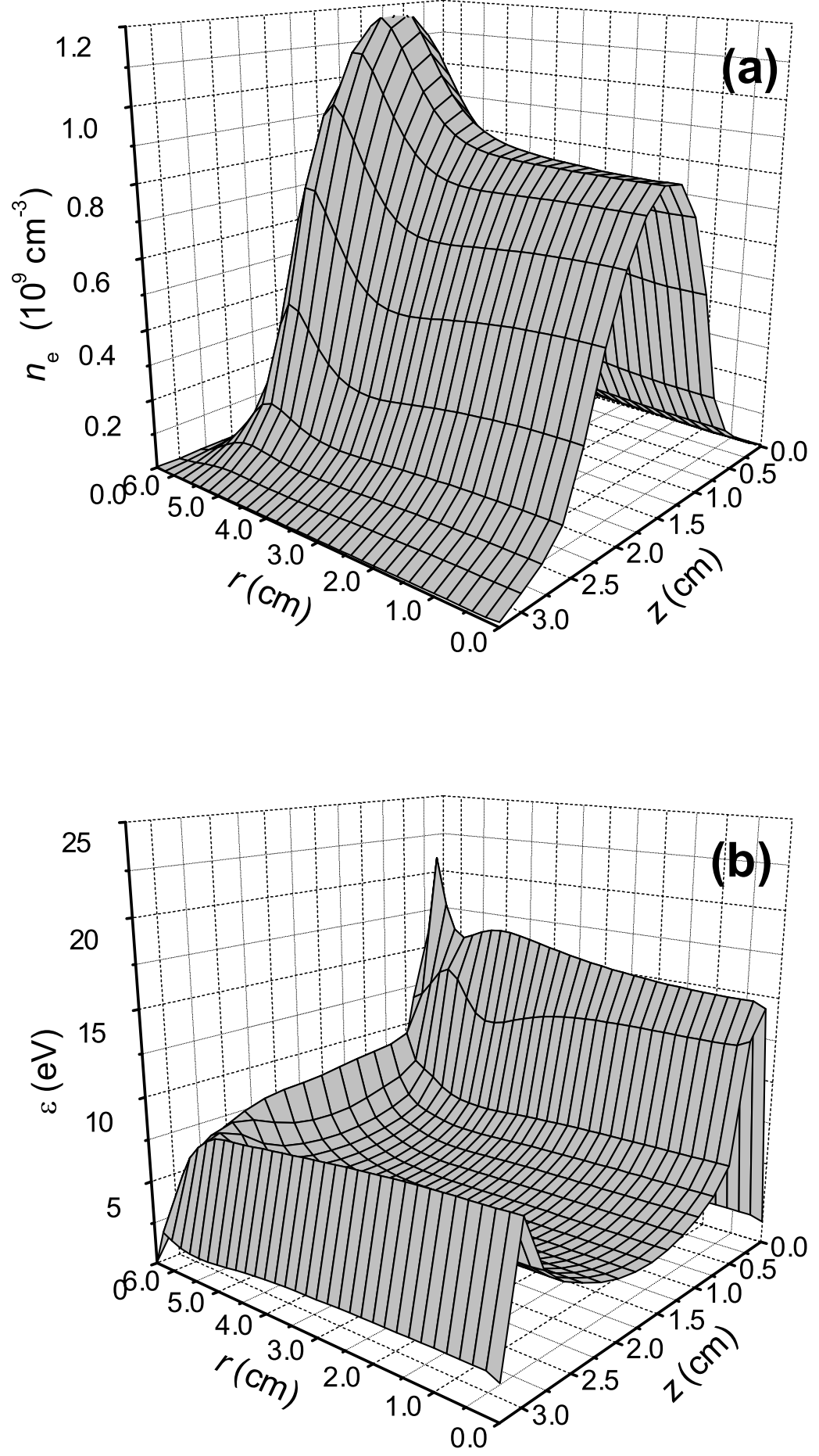

FIG. 1: A. Salabas, J. Appl. Phys. 

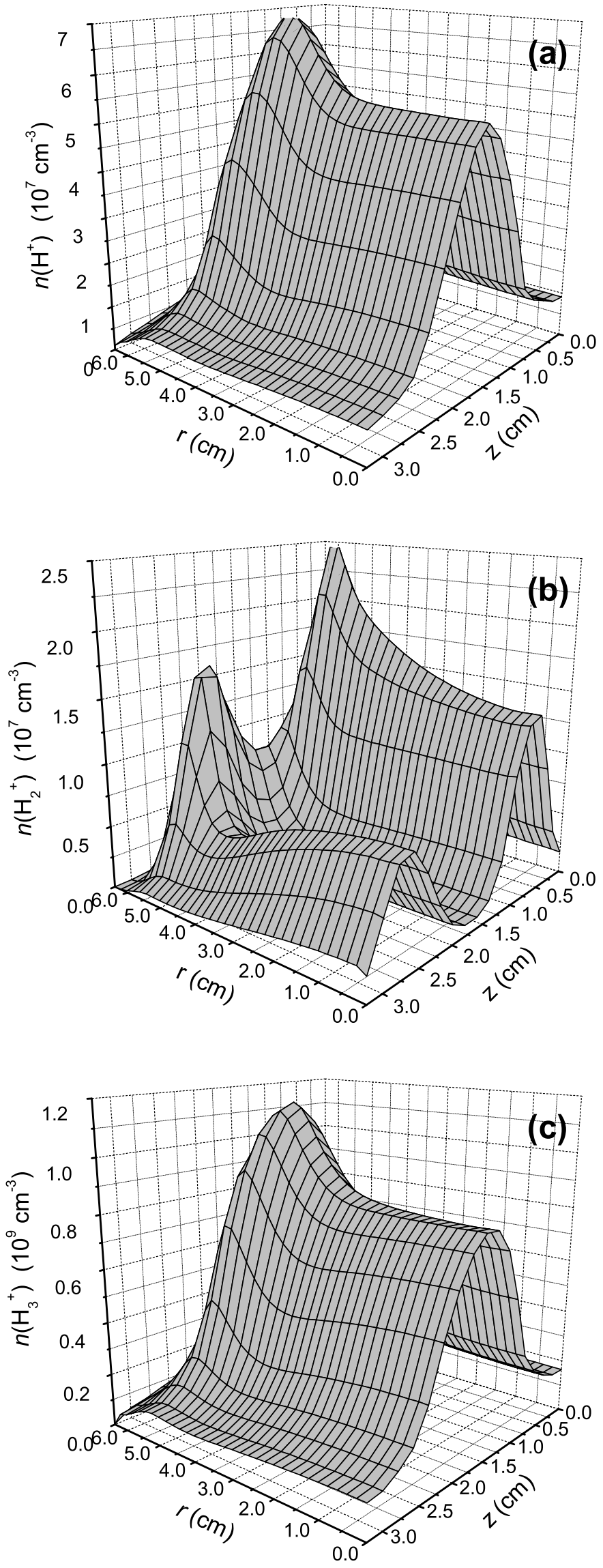

FIG. 2: A. Salabas, J. Appl. Phys. 

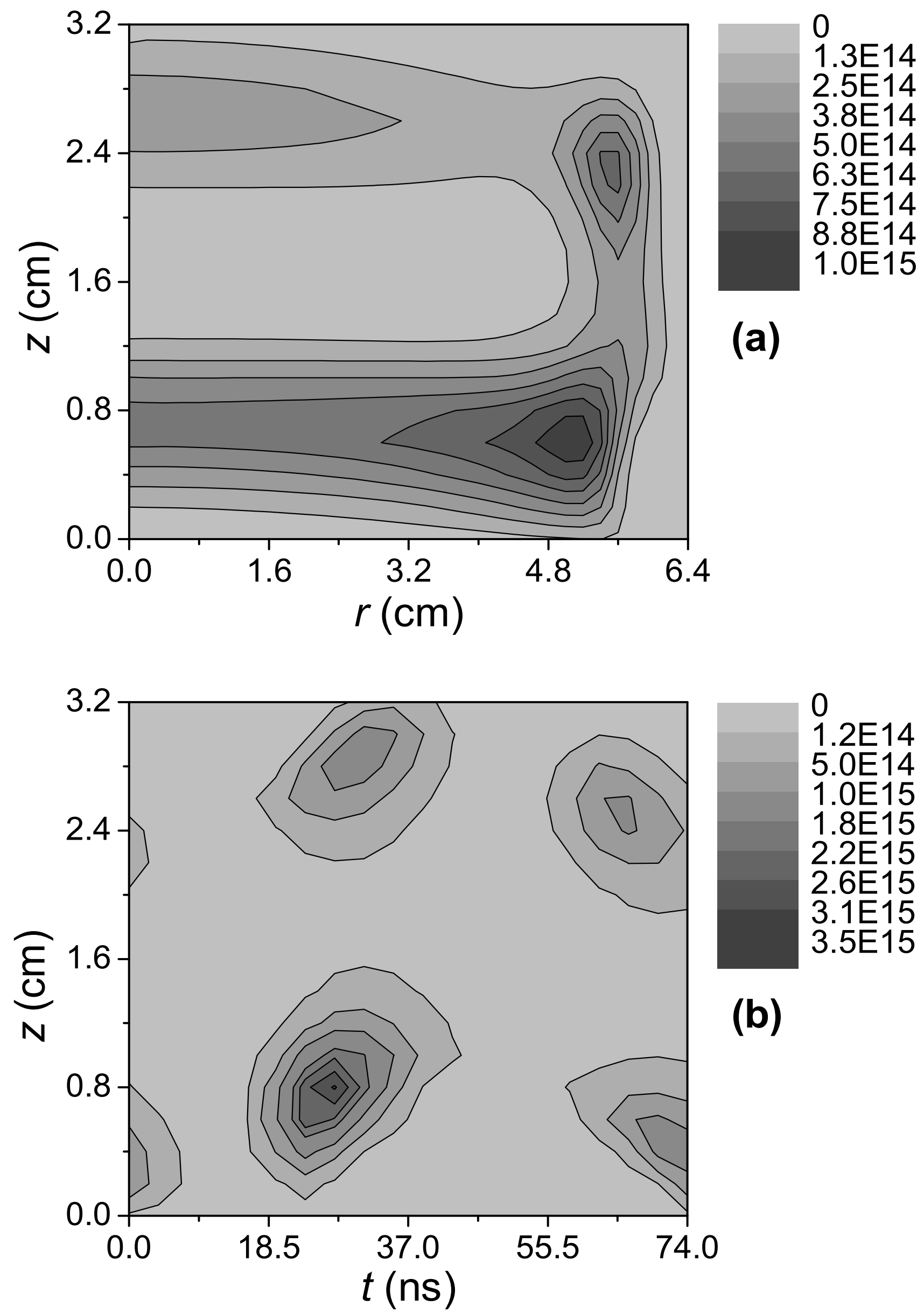

1.2E14 5.0E14 1.0E15 1.8E15 2.2E15 2.6E15 $3.1 \mathrm{E} 15$ $3.5 \mathrm{E} 15$ 

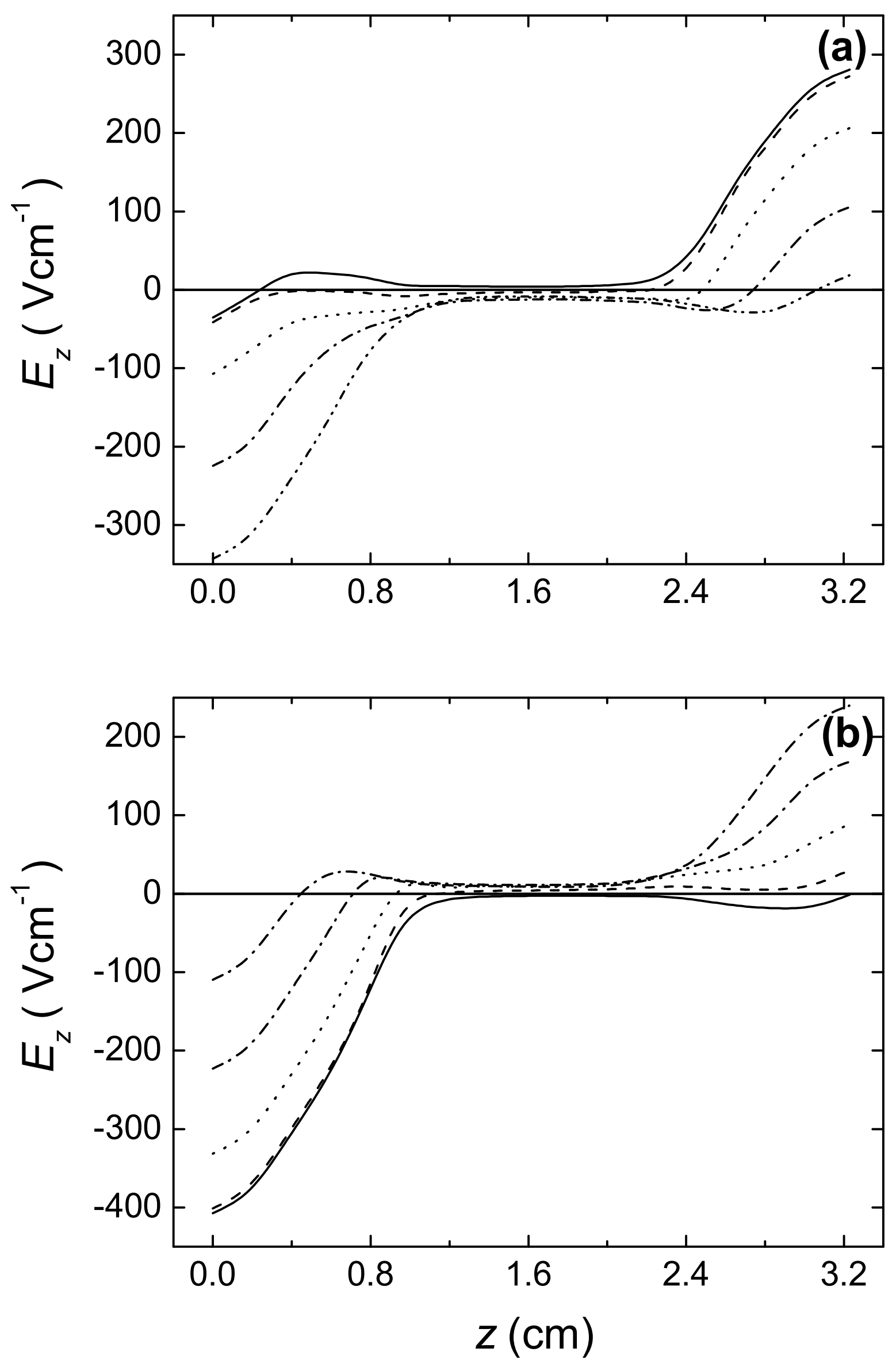

FIG. 4: A. Salabas, J. Appl. Phys. 


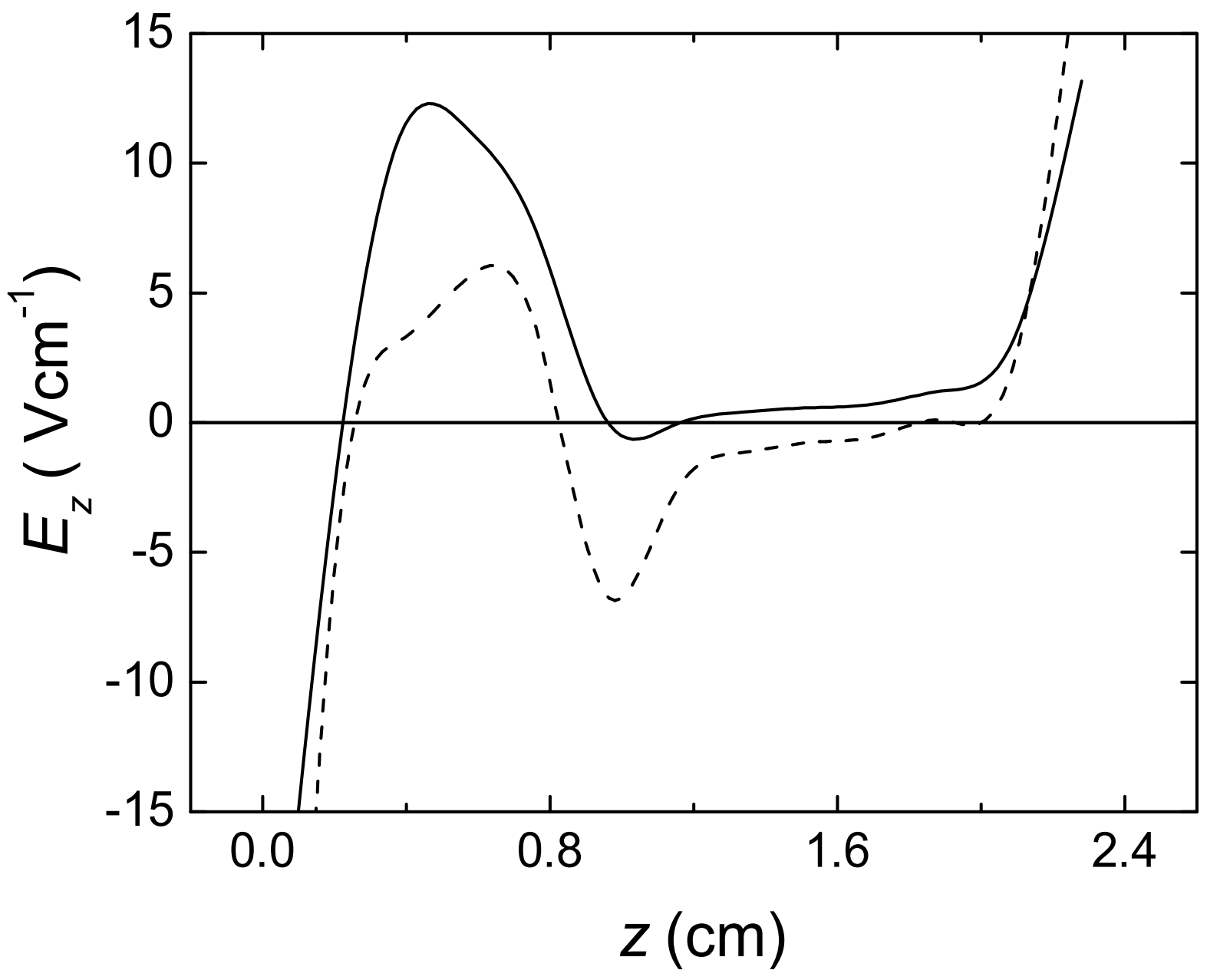

FIG. 5: A. Salabas, J. Appl. Phys. 


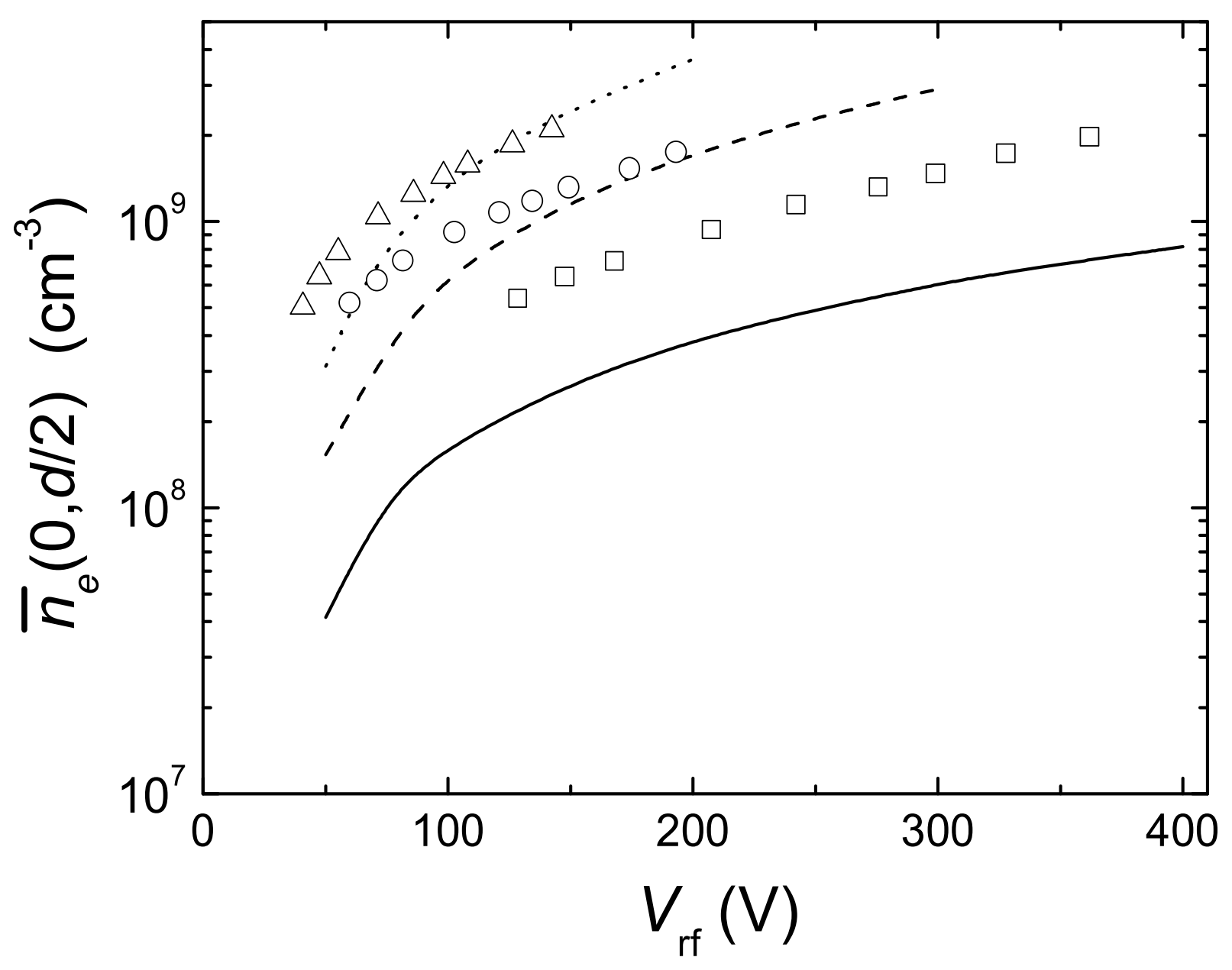

FIG. 6: A. Salabas, J. Appl. Phys. 


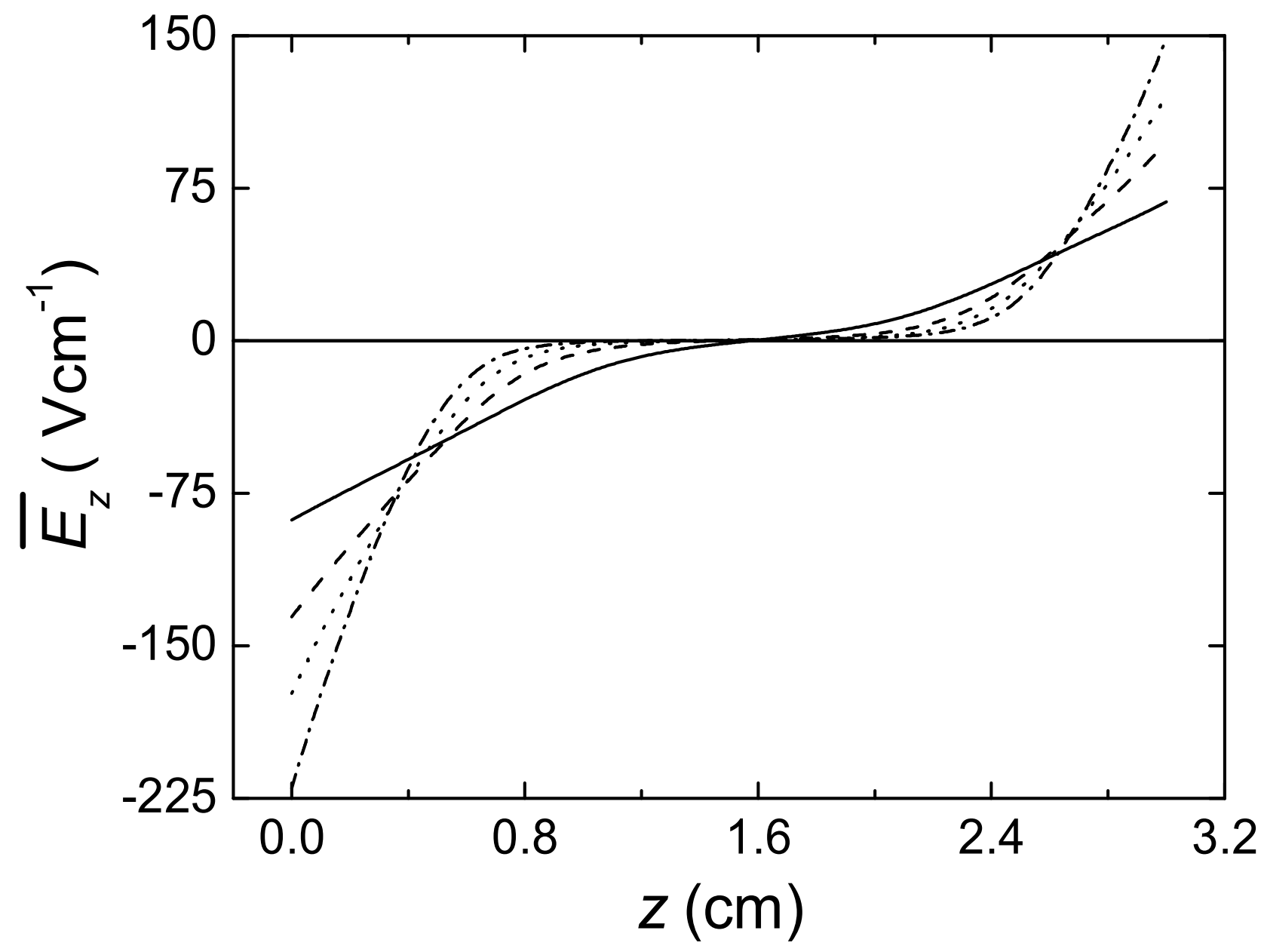

FIG. 7: A. Salabas, J. Appl. Phys. 

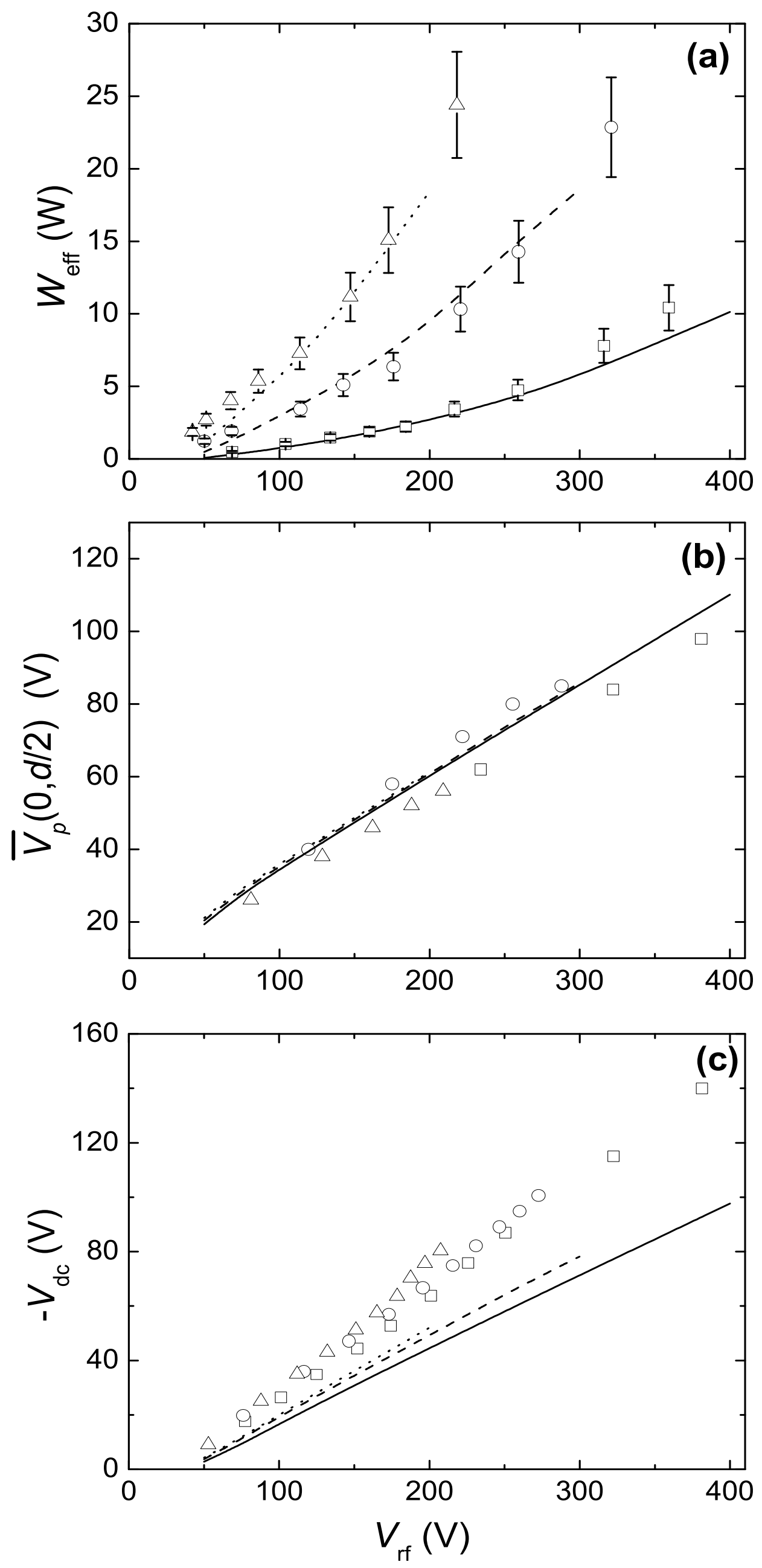

FIG. 8: A. Salabas, J. Appl. Phys. 


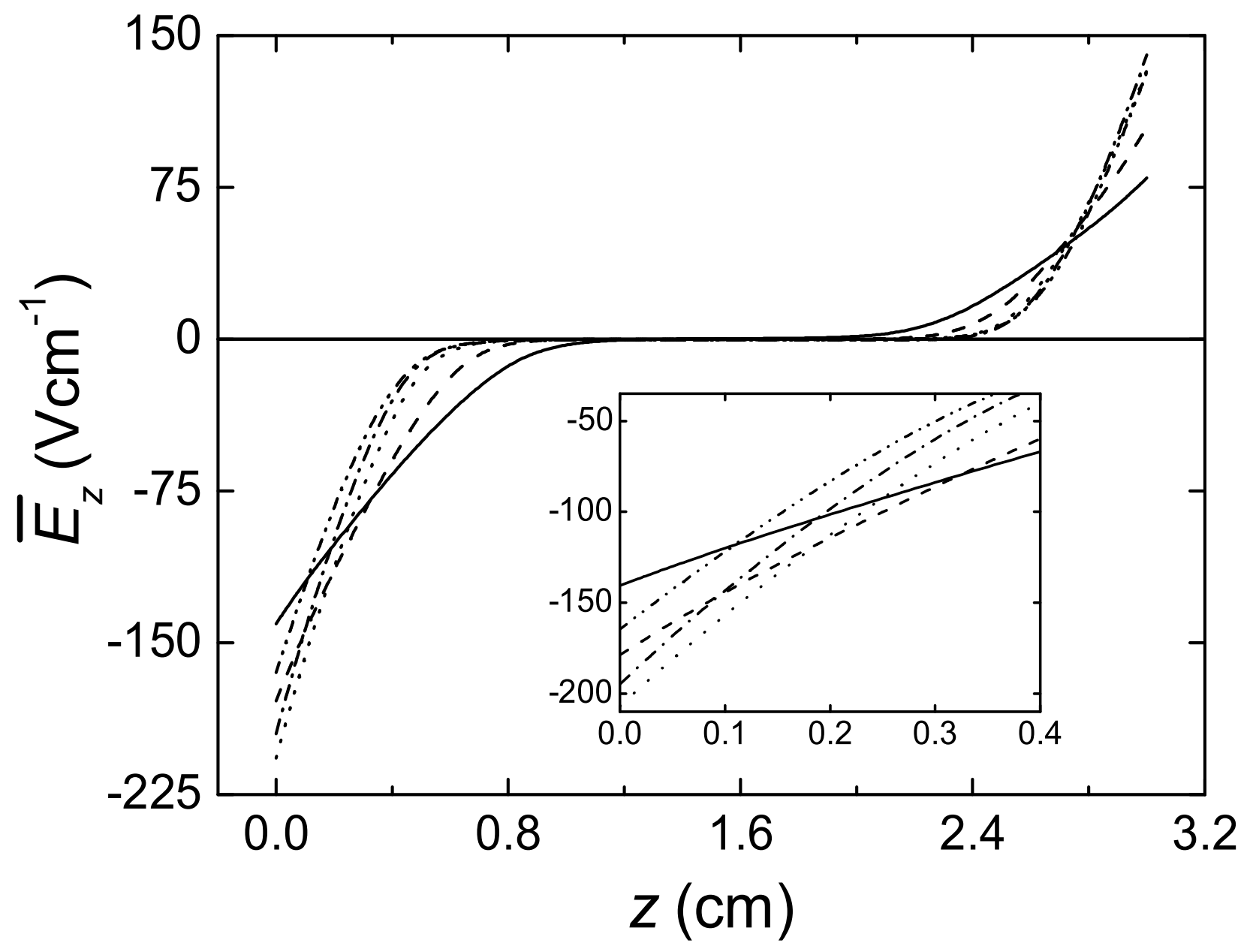

FIG. 9: A. Salabas, J. Appl. Phys. 


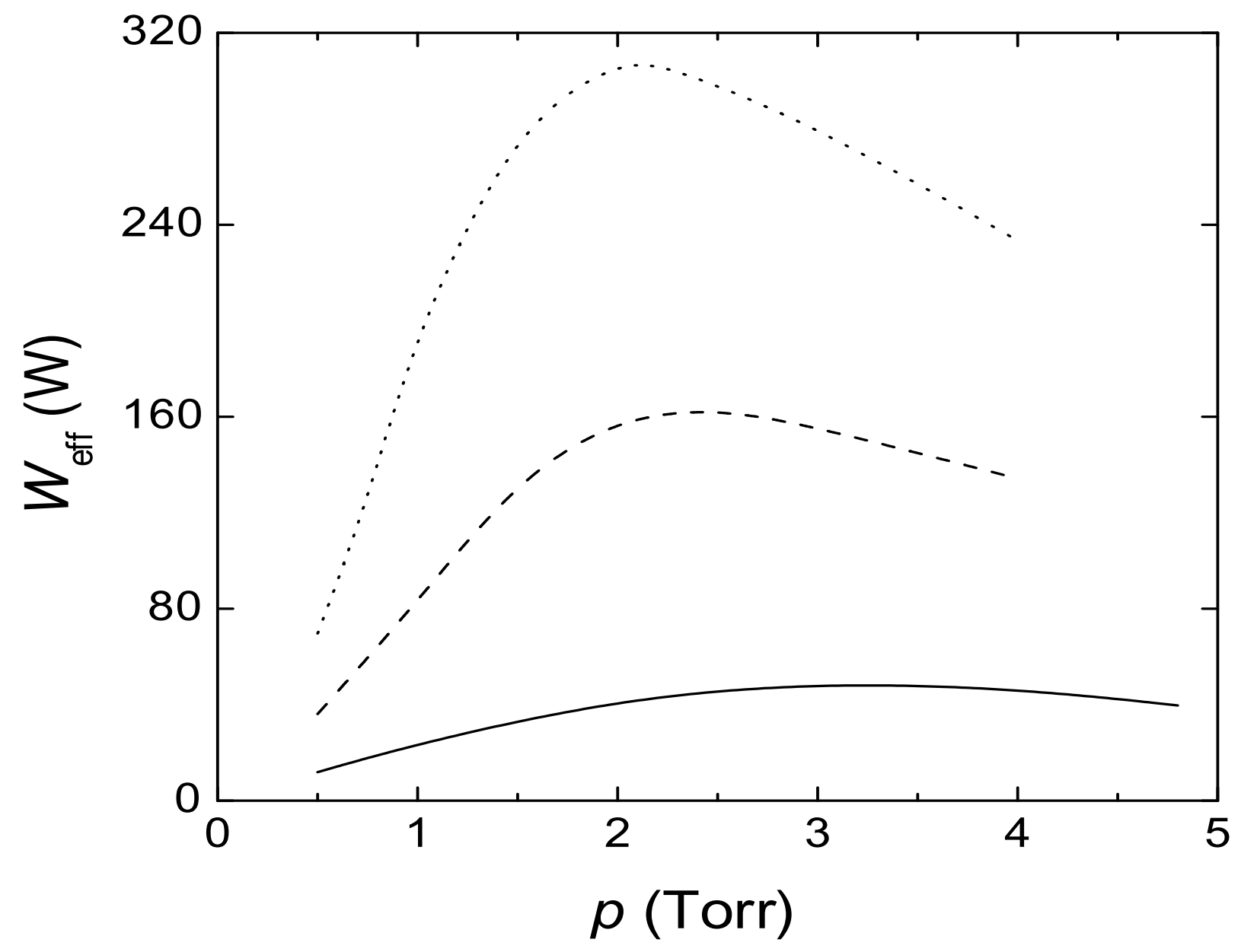

FIG. 10: A. Salabas, J. Appl. Phys. 

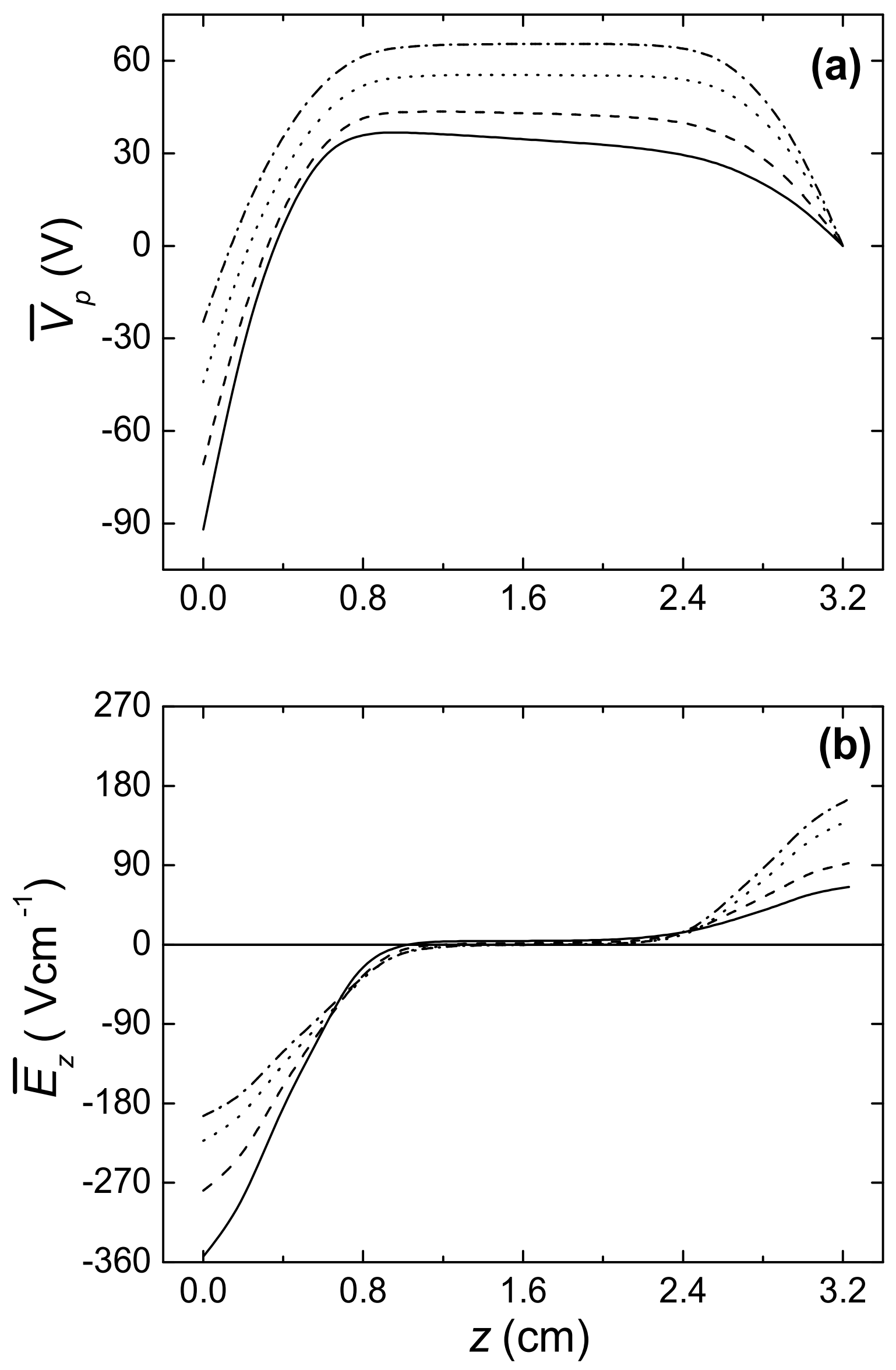

FIG. 11: A. Salabas, J. Appl. Phys. 

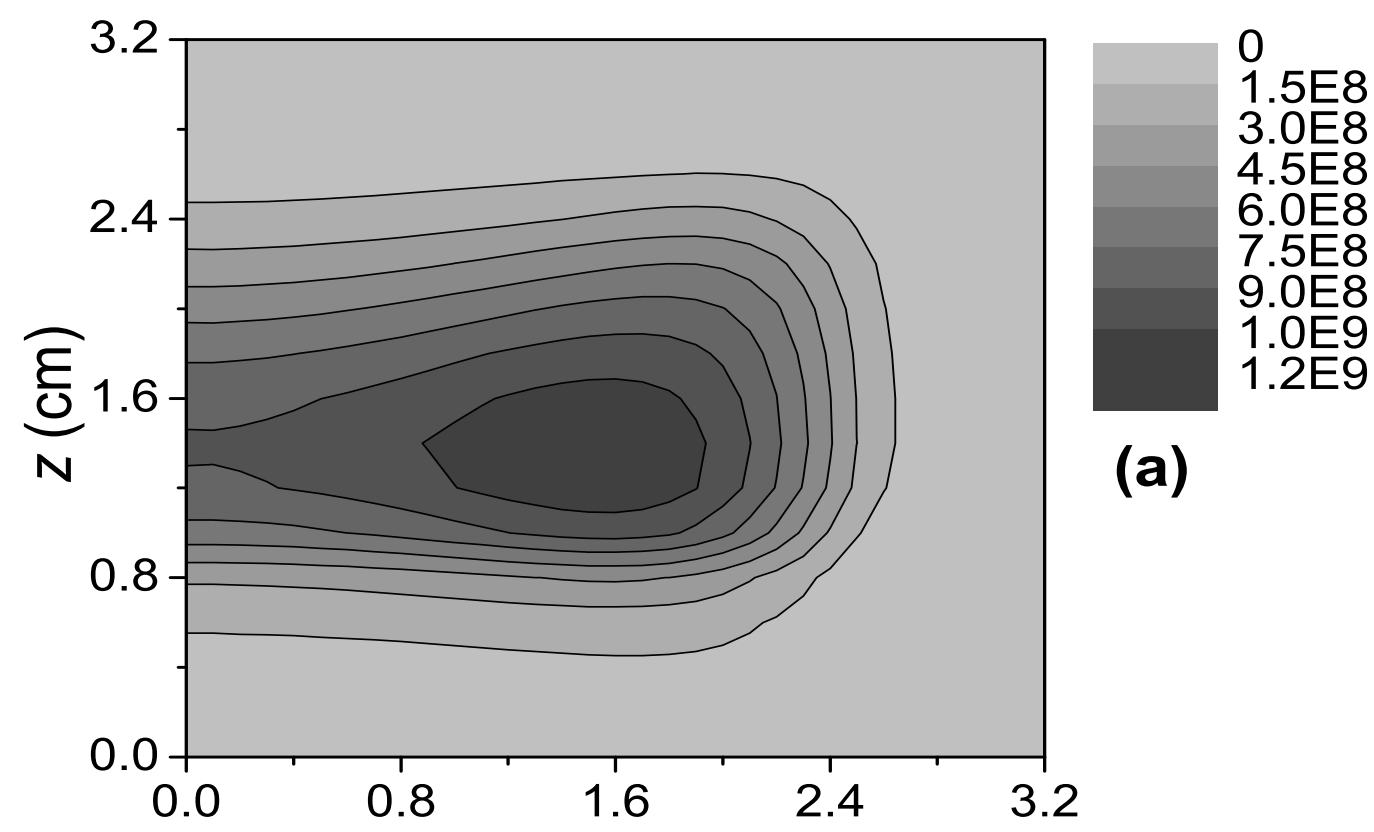

(a)
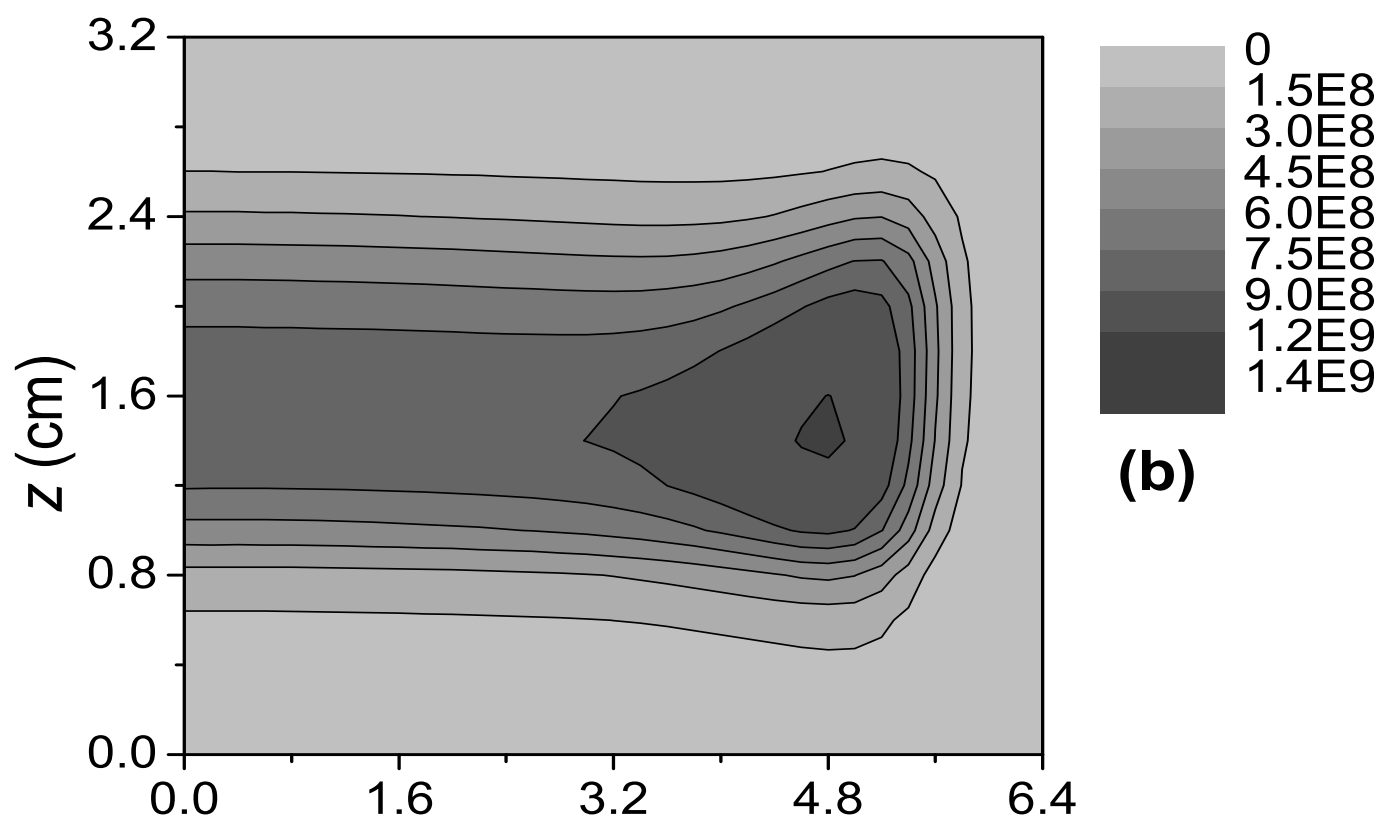

(b)

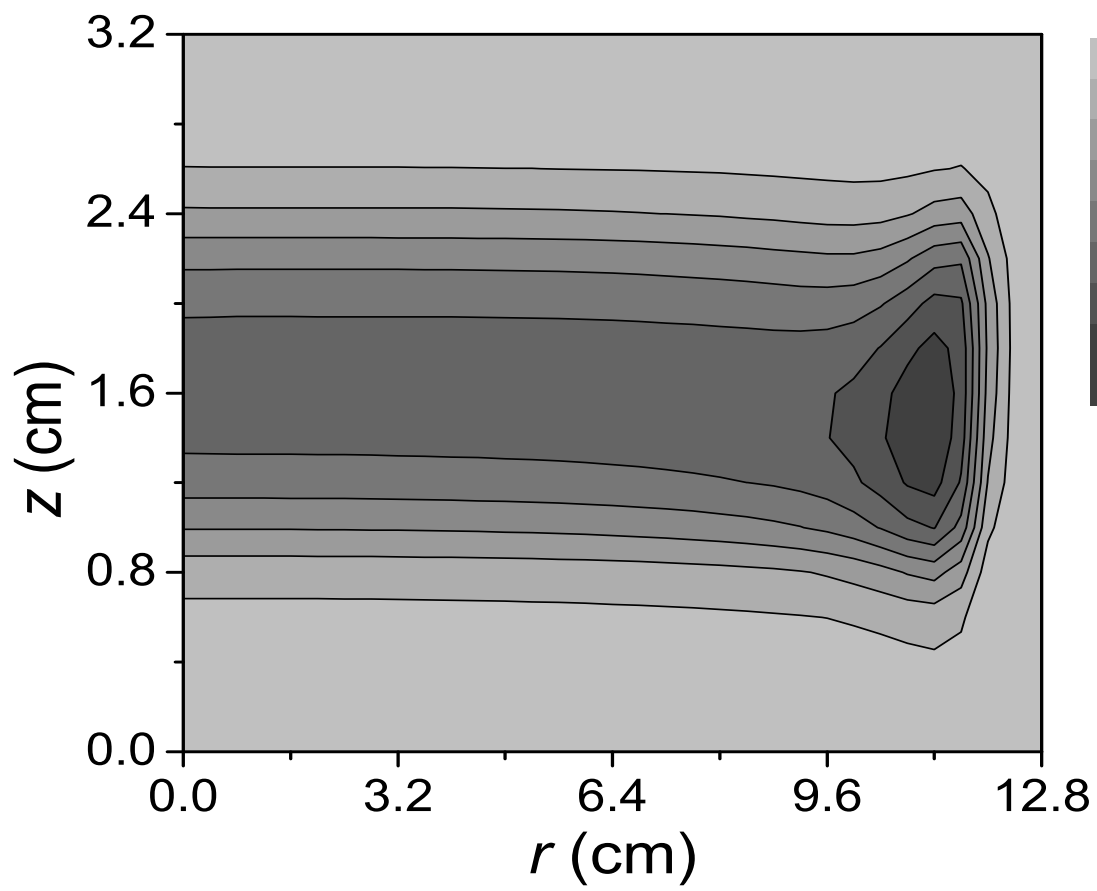

1.5E8 3.0E8 4.5E8 6.0E8 7.5E8 9.0E8 1.0E9 1.2E9

(c) 

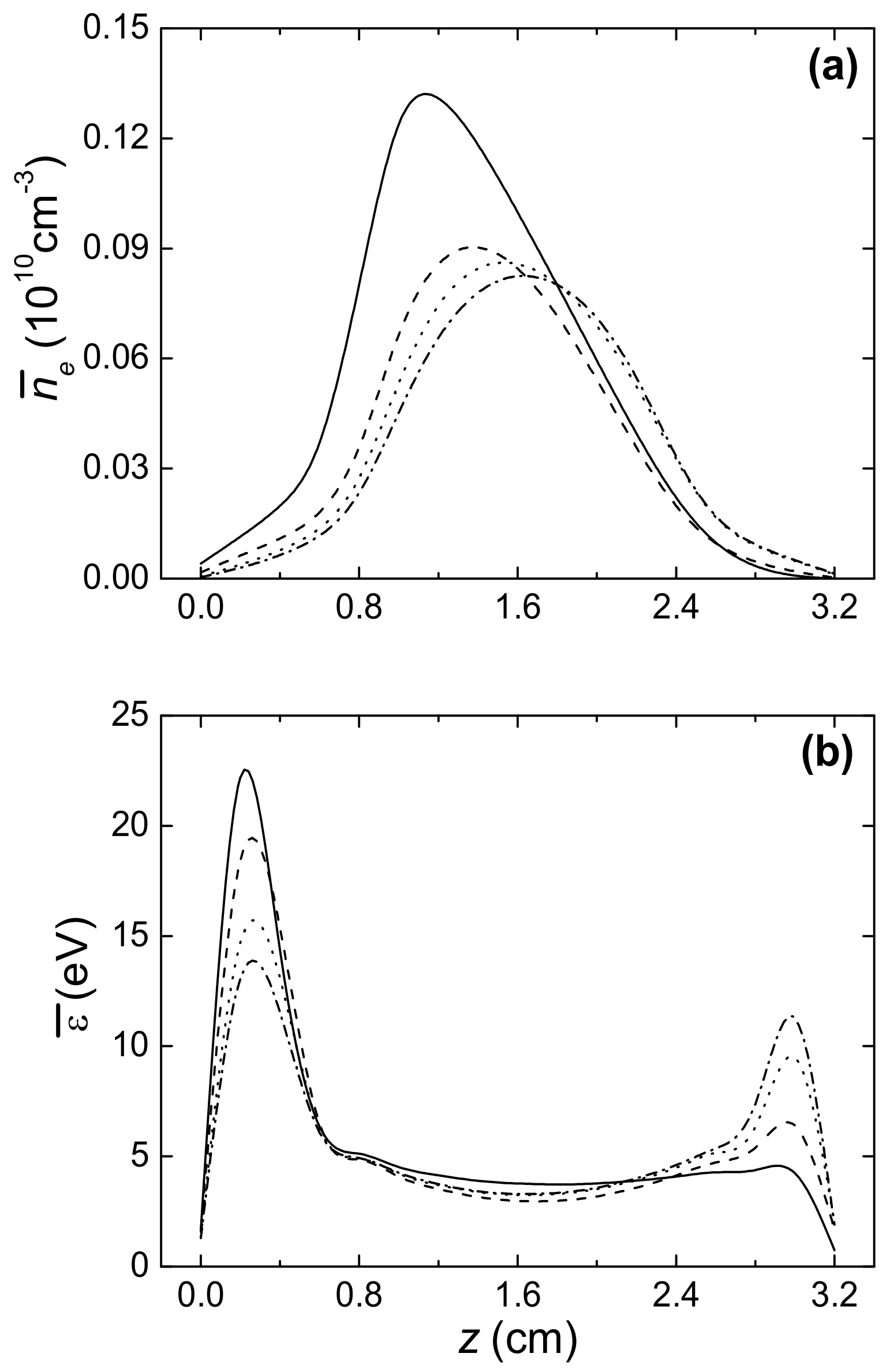

FIG. 13: A. Salabas, J. Appl. Phys. 

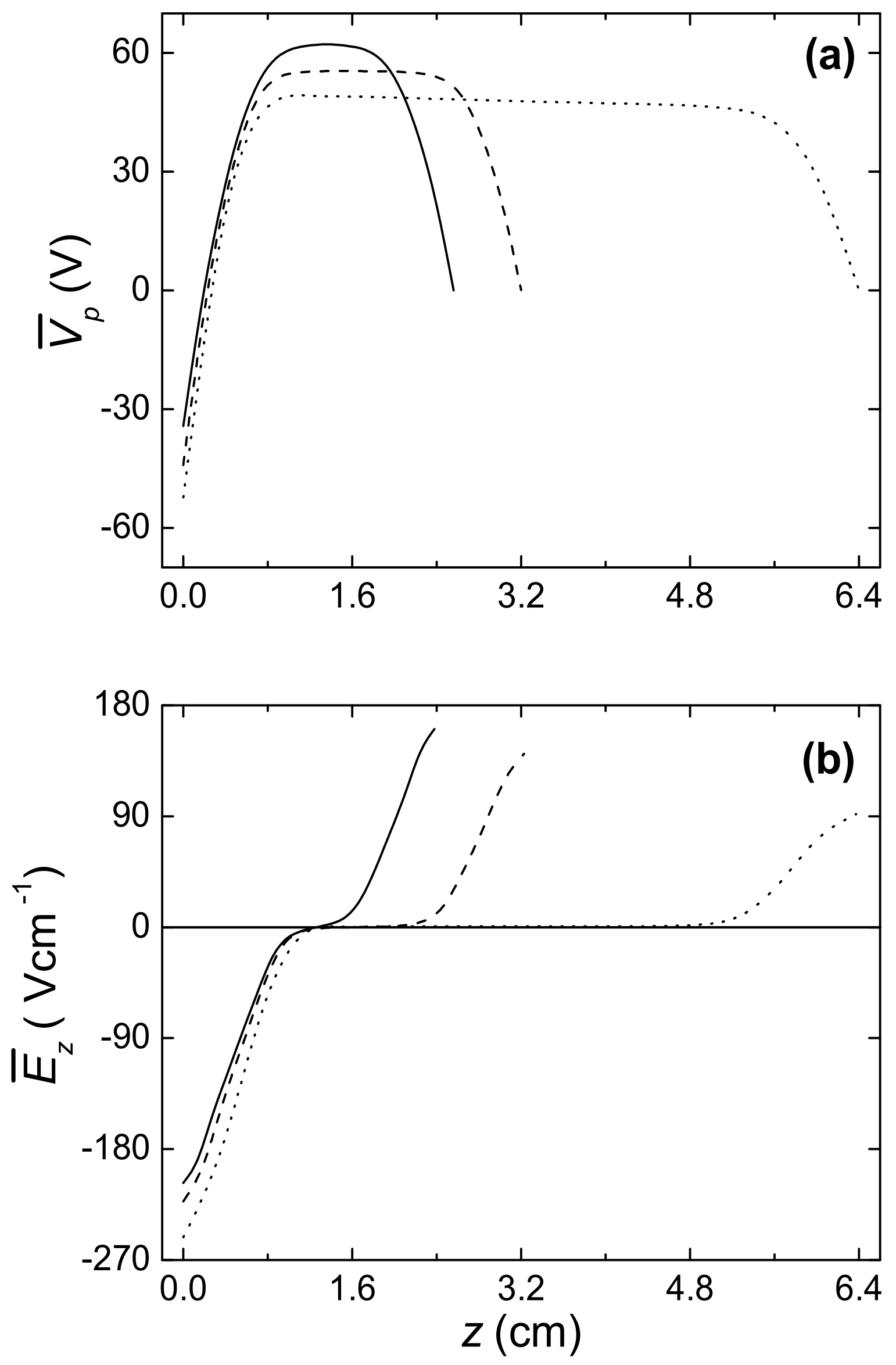

FIG. 14: A. Salabas, J. Appl. Phys. 

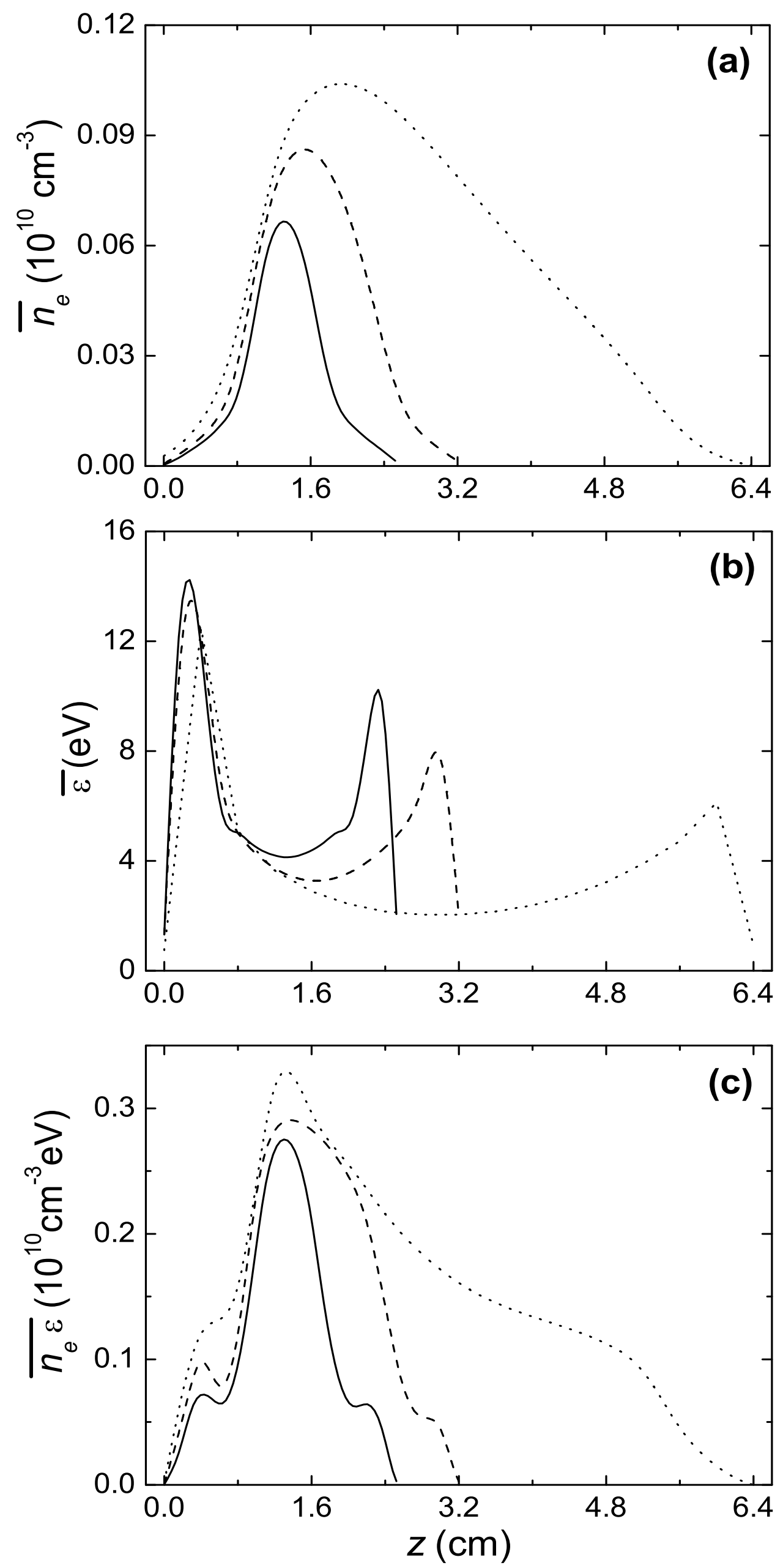

FIG. 15: A. Salabas, J. Appl. Phys. 

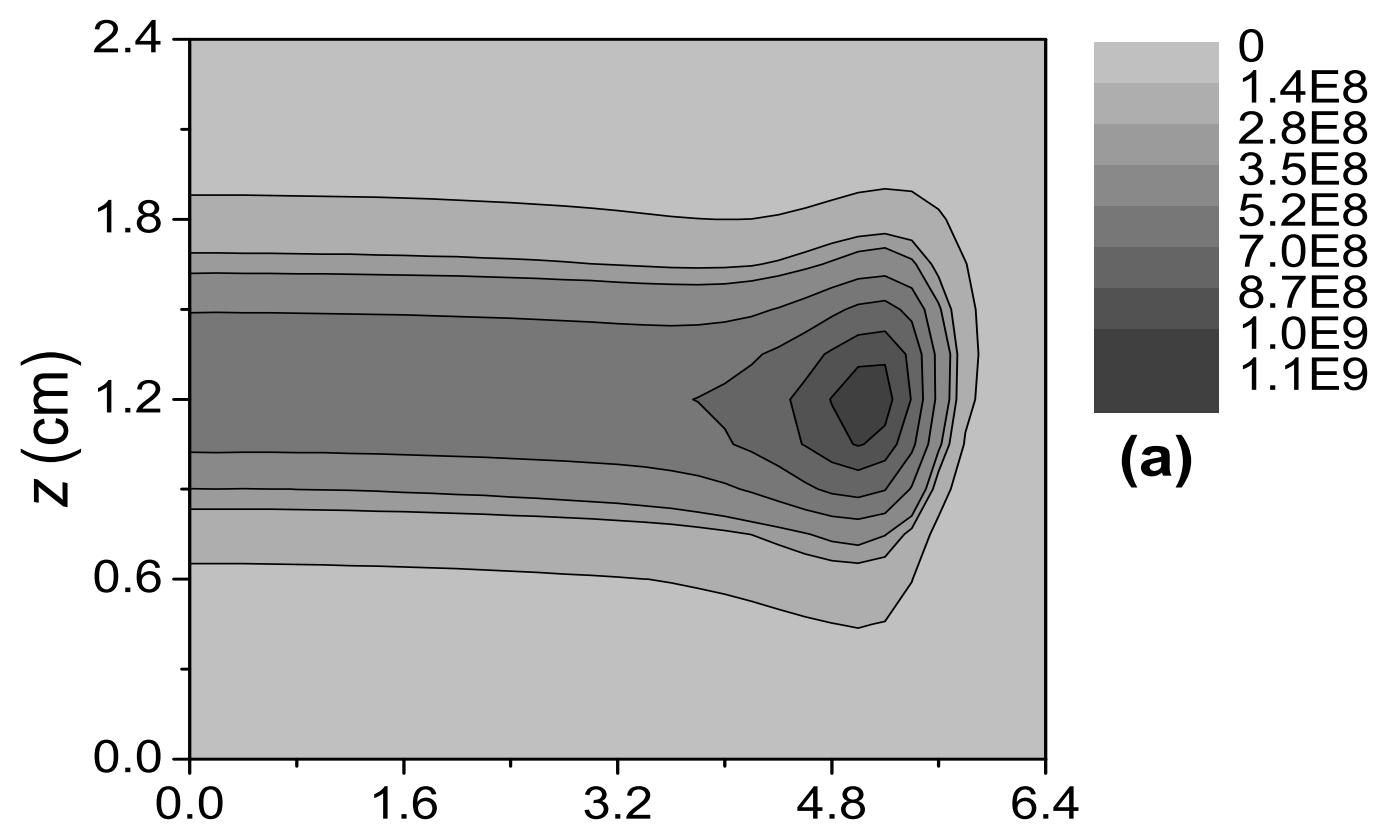

(a)
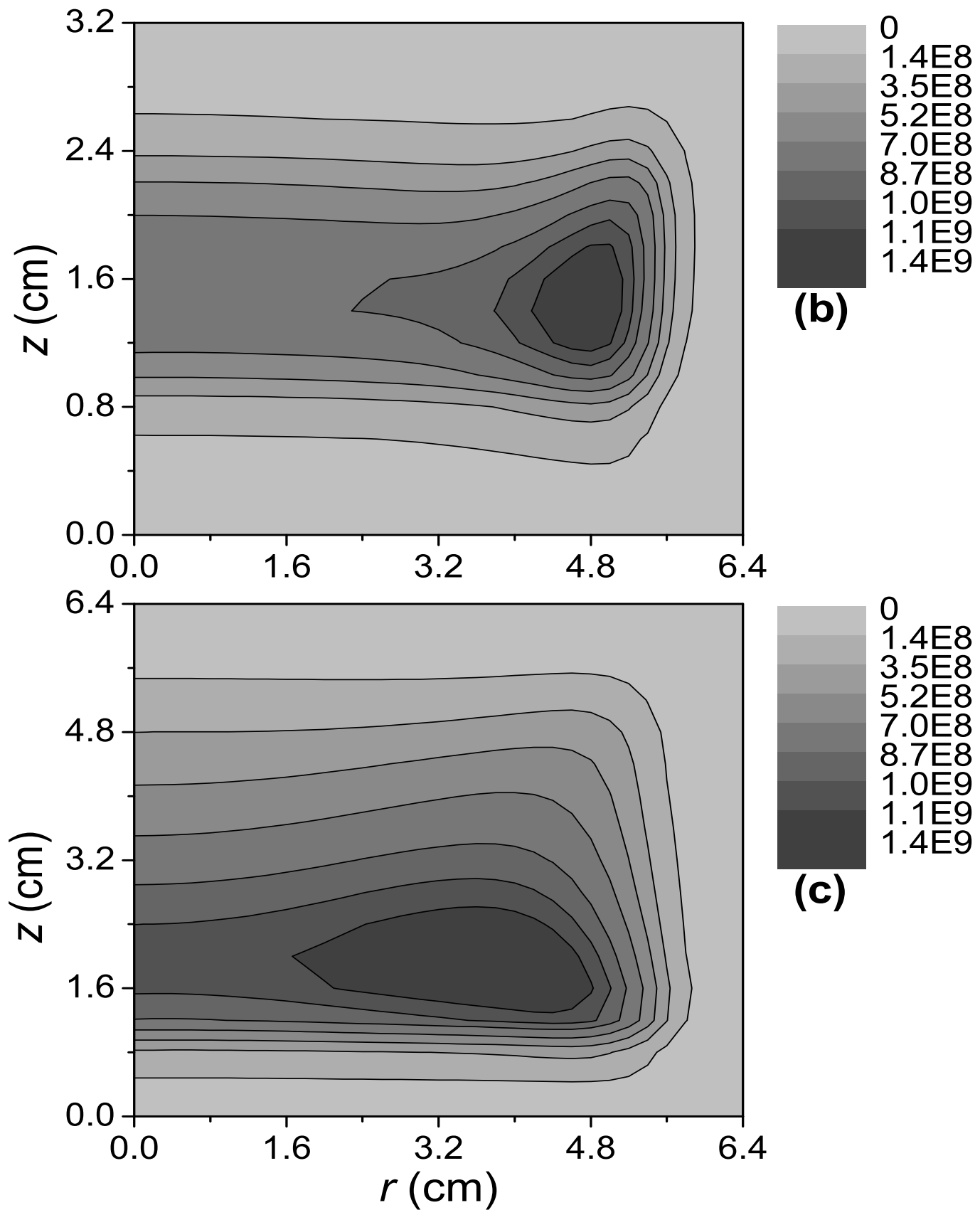

1.4E8 $3.5 \mathrm{E} 8$ $5.2 \mathrm{E} 8$ 7.0E8 8.7E8 $1.0 \mathrm{E} 9$ $1.1 \mathrm{E} 9$ 1.4E9

(c)

FIG. 16: A. Salabas, J. Appl. Phys. 

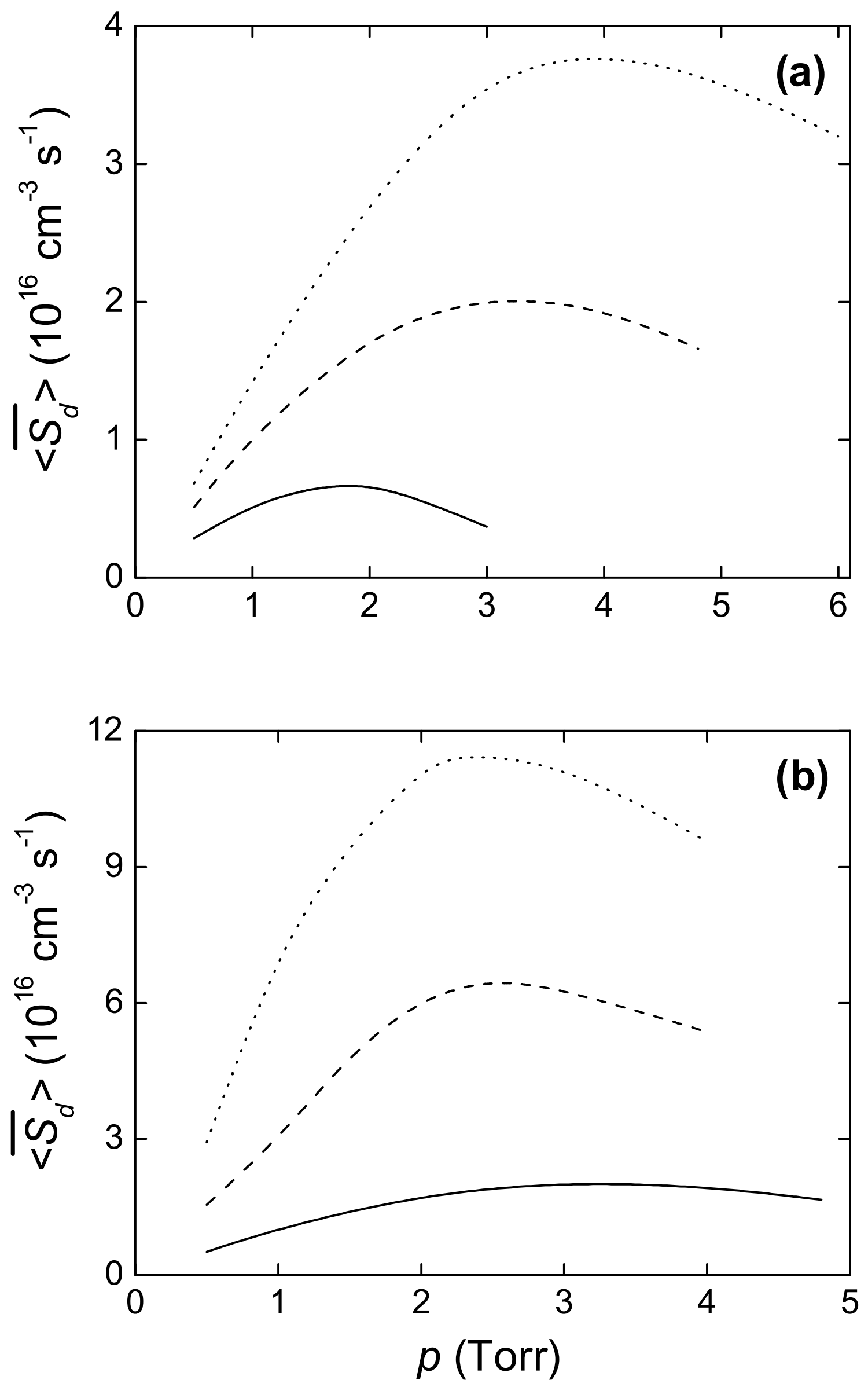

FIG. 17: A. Salabas, J. Appl. Phys. 

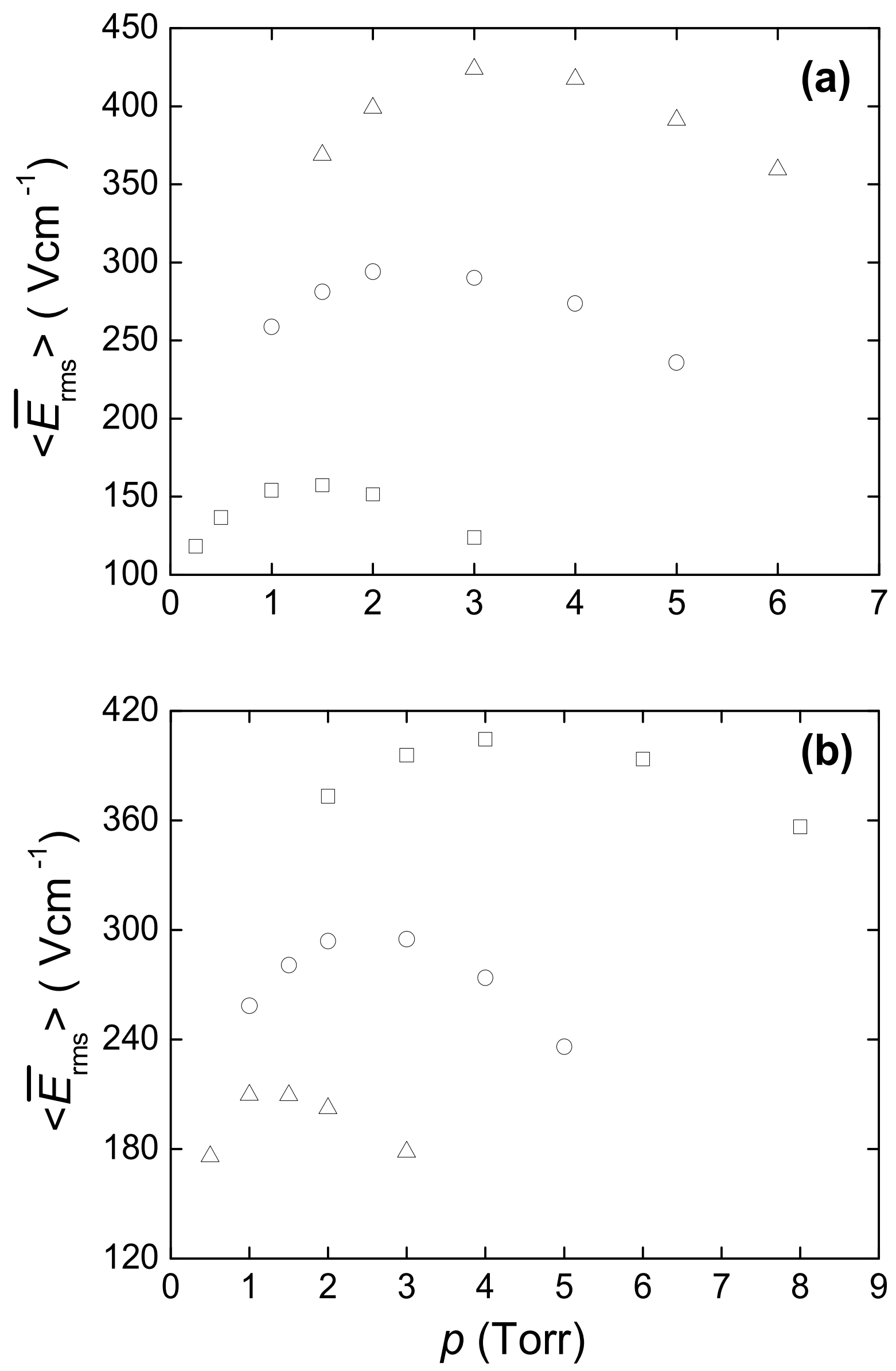

FIG. 18: A. Salabas, J. Appl. Phys. 


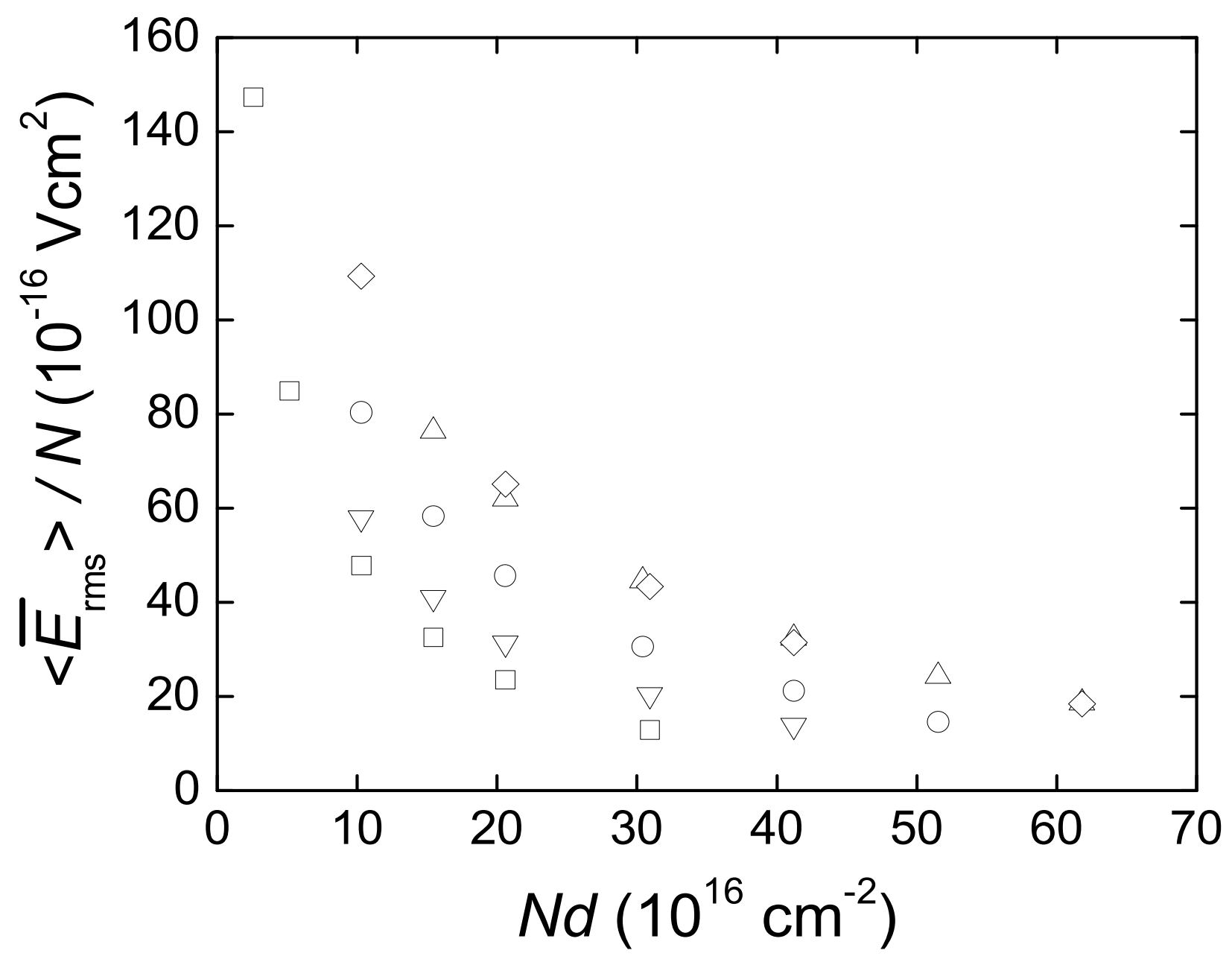

FIG. 19: A. Salabas, J. Appl. Phys. 


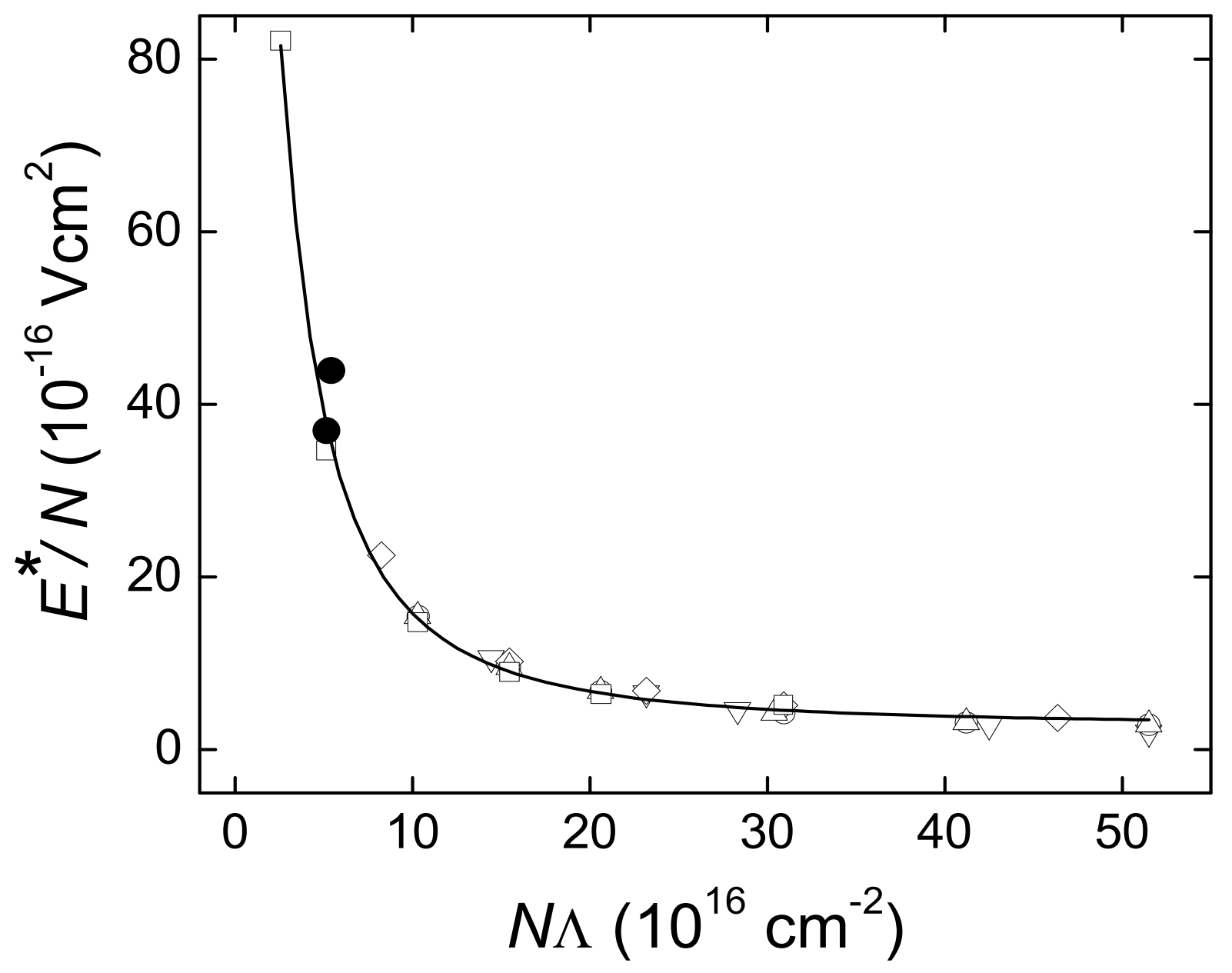

FIG. 20: A. Salabas, J. Appl. Phys. 